\title{
ON THE GENERICITY OF THE OBSERVABILITY OF CONTROLLED DISCRETE-TIME SYSTEMS
}

\author{
SABEuR AmmaR ${ }^{1}$ AND JeAn-Claude VivaldA ${ }^{1}$
}

\begin{abstract}
In this paper, we prove the genericity of the observability for discrete-time systems with more outputs than inputs.
\end{abstract}

Mathematics Subject Classification. 57R40, 93B07, 93B27, 93C55.

Received October 14, 2003.

\section{INTRODUCTION}

In this paper, we study the genericity of the observability for discrete-time controlled nonlinear systems such that:

$$
\left\{\begin{array}{c}
x_{k+1}=f\left(x_{k}, u_{k}\right) \\
y_{k}=h\left(x_{k}, u_{k}\right) \\
x_{k} \in X, u \in U, y_{k} \in \mathbb{R}^{p}
\end{array}\right.
$$

where:

- $X$ and $U$ are $C^{\infty}$ compact connected second-countable manifold with dimensions $n$ and $m$ respectively;

- $f: X \times U \rightarrow X$ is a parameterized diffeomorphism: that is to say, for every $u \in U$, the mapping $f(\cdot, u)$ is a $C^{\infty}$ diffeomorphism; we denote by Diff ${ }_{U}$ the set of all parameterized diffeomorphisms;

- $h: X \times U \rightarrow \mathbb{R}^{p}$ is a $C^{\infty}$ mapping.

To be more specific, we will introduce some notations; given $f \in \operatorname{Diff}_{\mathrm{U}}(X)$ and $h \in C^{\infty}\left(X \times U, \mathbb{R}^{p}\right)$, we denote by $u_{N}$ the finite sequence $\left(u_{0}, \ldots, u_{N-1}\right)$ of elements of $U$, and we define recursively $f^{k}\left(x, u_{k}\right)$ by

$$
\begin{aligned}
f^{1}\left(x, \underline{u_{1}}\right) & =f\left(x, u_{0}\right) \\
f^{k+1}\left(x, \underline{u_{k+1}}\right) & =f\left(f^{k}\left(x, \underline{u_{k}}\right), u_{k}\right) \quad \text { for } k \geq 1 .
\end{aligned}
$$

Let us recall the notion of observability investigated in this paper.

Definition 1. Two initial conditions $x_{0}$ and $\bar{x}_{0}$ and an input $u=\left(u_{k}\right)_{k \geq 0}$ being given, $x_{k}$ and $\bar{x}_{k}$ denote the points $x_{k}=f\left(x_{0}, \underline{u_{k}}\right)$ and $\bar{x}_{k}=f\left(\bar{x}_{0}, \underline{u_{k}}\right)$.

Keywords and phrases. Observability, nonlinear systems, discrete-time systems, transversality theory.

1 Inria-Lorraine \& UMR CNRS 7122 - ISGMP Bâtiment A, Université de Metz, Ile du Saulcy, 57045 Metz Cedex 01, France; ammar@loria.fr; vivalda@loria.fr

(c) EDP Sciences, SMAI 2005 
System (1) is said observable for input $u$ if for any initial conditions $x_{0} \neq \bar{x}_{0}$, there exists an index $k$ (possibly depending on the initial conditions) such that $x_{k} \neq \bar{x}_{k}$.

System (1) is said observable if it is observable for each input.

Below, we are introducing a stronger notion of observability. We consider the application $\Theta_{2 n+1}^{f, h}$ from $X \times U^{2 n+1}$ to $\mathbb{R}^{(2 n+1) p} \times U^{2 n+1}$ defined by

$$
\Theta_{2 n+1}^{f, h}\left(x, \underline{u_{2 n+1}}\right)=\left(h\left(x, u_{0}\right), h\left(f^{1}\left(x, \underline{u_{1}}\right), u_{1}\right), \ldots, h\left(f^{2 n}\left(x, \underline{u_{2 n}}\right), u_{2 n}\right), \underline{u_{2 n+1}}\right) .
$$

Notice that this application is the discrete-time analogous of the application $S \Phi_{k}^{\Sigma}$ defined in [6].

Definition 2. We will say that system (1) is strongly observable if the related application $\Theta_{2 n+1}^{f, h}$ defined above is one-to-one.

In this article, we prove that system (1) is generically strongly observable as long as $p>\operatorname{dim} U$; in other words any system such that (1) can be approximated by another strongly observable system.

On this subject, one has to mention first the important work from Gauthier and Kupka. In a first paper, with also Hammouri [3], the authors investigated the genericity of observability for uncontrolled continuoustime systems. This work was generalized by Gauthier and Kupka in [5,6] the authors proved the genericity of differential observability for systems with more outputs than inputs. As far as we are concerned by discrete-time systems, we have to cite several papers on the subject of the genericity of the observability: first, a paper written by Aeyels [2] in which the author considers uncontrolled continuous-time systems and their discretized. In this paper, the author introduced the notion of $P$-observability. The system

$$
\left\{\begin{array}{l}
\dot{x}=f(x) \\
y=h(x)
\end{array}\right.
$$

is said $P$-observable if, given a time $T>0$ and a finite subset $P$ of $[0, T]$, for every pair $(x, y)$ of distinct elements in $X^{2}$, there exists a $t_{i} \in P$ such that $h \circ \Phi_{t_{i}}(x) \neq h \circ \Phi_{t_{i}}(y)$ where $\Phi$ denotes the flow of $f$. One of the results in this paper is the proof of the existence of an open and dense set of vector fields such that, a vector field $f$ in this set being fixed, the subset of functions $h$ belonging to $C^{r}(X, \mathbb{R})$ such that the system $(f, h)$ is $P$-observable is open and dense in $C^{r}(X, \mathbb{R})$. This is true for almost any finite subset $P$ of $(2 \operatorname{dim} X+1)$ points in $[0, T]$.

To an uncontrolled discrete-time systems such that

$$
\left\{\begin{array}{l}
x_{k+1}=f\left(x_{k}\right) \\
y_{k}=h\left(x_{k}\right) \\
x_{k} \in M, \text { compact manifold, } y_{k} \in \mathbb{R}
\end{array}\right.
$$

is attached a map analogous to the map $\Theta_{2 n+1}^{f, h}$ defined above: consider

$$
\begin{aligned}
\Phi: M & \longrightarrow \mathbb{R}^{2 n+1} \\
x & \longmapsto\left(h(x), h \circ f(x), \ldots, h \circ f^{2 n}(x)\right)
\end{aligned}
$$

where $n$ is the dimension of manifold $M$. In [10], the proof that, generically, $\Phi$ is an embedding is sketched while in [8] and [11], the same result is proved in greater detail.

In the case of controlled discrete-time systems, in article [9], the authors investigate controlled discrete-time systems and obtain some results which are similar (but not identical) to the one presented here.

Before going straight to the point, we want to add some words about the fact that the observation function $h$ depends on $u$. This situation is not common in automatic control theory, but the opposite assumption leads to clumsy statements. Nevertheless, in the conclusion we roughly explain how the result of genericity can be proved for systems where $h$ does not depend on $u$. The paper is organized as follows: in the next section, 
some facts from transversality theory are recalled, in Section 3, the main result is stated together with some definitions and lemmas; in Section 4, our result is proved through the demonstrations of three lemmas and, finally, a conclusion is made in Section 5.

\section{SOME FACTS FROM TRANSVERSALITY THEORY}

In this section we recall some theorems from differential topology which will be intensively used in the proof of the main result of this paper. For details on the $C^{\infty}$ Whitney topology, the reader is referred to the book "Stable Mappings and their Singularities" [7].

If $X$ and $Y$ are two smooth manifolds, $J^{k}(X, Y)$ will denote, as usual, the set of $k$-jets from $X$ to $Y$, $\alpha: J^{k}(X, Y) \rightarrow X$ is the source map and $\beta: J^{k}(X, Y) \rightarrow Y$ the target map; moreover we denote by $C^{r}(X, Y)$ $(1 \leq r \leq+\infty)$ the set of $C^{r}$ maps from $X$ to $Y$. If $f$ is in $C^{\infty}(X, Y) j^{k} f$ denotes the $k$-jet of $f$. Recall that the set $C^{\infty}(X, Y)$ endowed with the Whitney topology is a Baire space and so every residual set of $C^{\infty}(X, Y)$ (i.e. every countable intersection of open dense subsets) is dense.

The notion of transversality is of paramount importance for our purpose and we recall below its definition.

Definition 3. Let $f$ be a smooth mapping between two smooth manifolds $X$ and $Y, W$ a submanifold of $Y$ and $x$ a point in $X$. We will say that $f$ intersects $W$ transversely at $x$ if either

- $f(x) \notin W$, or

- $f(x) \in W$ and $T_{f(x)} Y=T_{f(x)} W+d f_{x}\left(T_{x} X\right)$,

$T_{x} X$ denoting the tangent space to $X$ at $x$ and $d f_{x}$ the Jacobian of $f$ at $x$. We will say that $f$ intersects $W$ transversely if it intersects $W$ transversely at $x$ for all $x$ in $W$. We will use of the symbol $\pitchfork$ to denote the transversality.

The following theorem states a result of genericity [7].

Theorem 1 (Thom transversality theorem). Let $X$ and $Y$ be smooth manifold and $W$ a submanifold of $J^{k}(X, Y)$ and let

$$
T_{W}=\left\{f \in C^{\infty}(X, Y) \mid j^{k} f \pitchfork W\right\}
$$

Then $T_{W}$ is a residual subset of $C^{\infty}(X, Y)$ in the $C^{\infty}$ topology. Moreover, if $W$ is closed, then $T_{W}$ is open.

The following result generalizes the above theorem to multijet spaces. We first define the set $X^{(s)}=$ $\left\{\left(x_{1}, \ldots, x_{s}\right) \in X^{s} \mid x_{i} \neq x_{j}\right.$ for $\left.1 \leq i<j \leq s\right\}$ and the mapping

$$
\begin{aligned}
\alpha^{s}:\left(J^{k}(X, Y)\right)^{s} & \longrightarrow X^{s} \\
\left(\sigma_{1}, \ldots, \sigma_{s}\right) & \longmapsto\left(\alpha\left(\sigma_{1}\right), \ldots, \alpha\left(\sigma_{s}\right)\right)
\end{aligned}
$$

and we let $J_{s}^{k}(X, Y)=\left(\alpha^{s}\right)^{-1}\left(X^{(s)}\right), J_{s}^{k}(X, Y)$ is a submanifold of $\left(J^{k}(X, Y)\right)^{s}$.

For $f \in C^{\infty}(X, Y)$, we can define

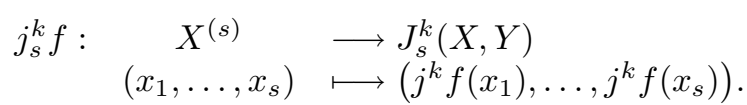

Theorem 2 (Multijet transversality theorem). Let $W$ be a submanifold of $J_{s}^{k}(X, Y)$ and let

$$
T_{W}=\left\{f \in C^{\infty}(X, Y) \mid j_{s}^{k} f \pitchfork W\right\}
$$

Then $T_{W}$ is a residual subset of $C^{\infty}(X, Y)$ in the $C^{\infty}$ topology. Moreover, if $W$ is compact, then $T_{W}$ is open.

We will use also a transversality theorem due to Abraham [1]. Let $\mathcal{A}, X$ and $Y$ be $C^{r}$ manifolds and $\rho$ a map from $\mathcal{A}$ to $C^{r}(X, Y)$. 
For $a \in \mathcal{A}$, we write $\rho_{a}$, the $C^{r}$ map:

$$
\begin{aligned}
\rho_{a}: X & \longrightarrow Y \\
x & \longmapsto \rho_{a}(x)=\rho(a)(x)
\end{aligned}
$$

and we say that $\rho$ is a $C^{r}$ representation if the evaluation map:

$$
\begin{aligned}
\mathrm{ev}_{\rho}: \mathcal{A} \times X & \longrightarrow Y \\
(a, x) & \longmapsto \rho_{a}(x)=\rho(a)(x)
\end{aligned}
$$

is a $C^{r}$ map from $\mathcal{A} \times X$ to $Y$.

Theorem 3 (Abraham transversal density theorem). Let $\mathcal{A}, X, Y$ be $C^{r}$ manifolds, $\rho: \mathcal{A} \rightarrow C^{r}(X, Y)$ a $C^{r}$ representation, $W \subset Y$ a submanifold (not necessarily closed), and $\mathrm{ev}_{\rho}: \mathcal{A} \times X \rightarrow Y$ the evaluation map. Define $\mathcal{A}_{W} \subset \mathcal{A}$ by:

Assume that:

$$
\mathcal{A}_{W}=\left\{a \in \mathcal{A} \mid \rho_{a} \pitchfork W\right\} .
$$

(1) $X$ has a finite dimension $n$ and $W$ has a finite codimension $q$ in $Y$;

(2) $\mathcal{A}$ and $X$ are second countable;

(3) $r>\max (0, n-q)$;

(4) $\operatorname{ev}_{\rho} \pitchfork W$.

Then $\mathcal{A}_{W}$ is residual in $\mathcal{A}$.

Notice that manifold $\mathcal{A}$ is not necessarily finite dimensional; it may be a Banach space or an open subset of a Banach space.

Finally, we will need the following theorem that can also be found in [1].

Theorem 4 (Openness of transversal intersection). Let $\mathcal{A}, X$ and $Y$ be $C^{r}$ manifolds with $X$ finite dimensional, $W \subset Y$ a closed $C^{r}$ submanifold, $K$ a compact subset of $X$, and $\rho: \mathcal{A} \rightarrow C^{r}(X, Y)$ a $C^{r}$ representation. Then the subset $\mathcal{A}_{K W} \subset \mathcal{A}$ defined by

$$
\mathcal{A}_{K W}=\left\{a \in \mathcal{A} \mid \rho_{a} \pitchfork_{x} W \text { for } x \in K\right\}
$$

is open.

\section{MAin RESUlt}

We state here our main result and some lemmas used in the proof of our theorem. Our framework is the set $\operatorname{Diff}_{\mathrm{U}}(X) \times C^{\infty}\left(X \times U, \mathbb{R}^{p}\right)$ equipped with the Whitney topology; obviously $\operatorname{Diff}_{\mathrm{U}}(X)$ is open in $C^{\infty}(X \times U, X)$ for this topology. In the theorem below, we assume that $\operatorname{dim} U<p$.

Theorem 5. The set of applications $(f, h) \in \operatorname{Diff}_{\mathrm{U}}(X) \times C^{\infty}\left(X \times U, \mathbb{R}^{p}\right)$ such that the mapping $\Theta_{2 n+1}^{f, h}$ is one to one, contains a set which is residual in $\operatorname{Diff}_{\mathrm{U}}(X) \times C^{\infty}\left(X \times, \mathbb{R}^{p}\right)$ equipped with the Whitney topology.

For the proof, we need the Abraham's theorem [1]. Notice that in the continuous-time case, the set of pairs $(f, h)$ (with $f$ a parameterized vector field) is a Banach space for the $C^{r}$ topology $(r<+\infty)$ but this is not the case for the set of pairs $(f, h)$ where $f$ is a parameterized diffeomorphism. So, it is not possible to copy directly the reasoning of [5]. The proof of this theorem will be somewhat awkward and will be based on several technical lemmas. Before stating these lemmas, we describe below our global strategy.

Suppose that $\mathscr{P}_{1}(f, h)$ and $\mathscr{P}_{2}(f, h)$ are two properties depending on $(f, h) \in \operatorname{Diff}_{\mathrm{U}}(X) \times C^{\infty}\left(X \times U, \mathbb{R}^{p}\right)$ whose conjunction is equivalent to the injectivity of $\Theta_{2 n+1}^{f, h}$. In Lemmas 1 and 2, we will prove that the set

$$
E_{1}=\left\{(f, h) \in \operatorname{Diff}_{\mathrm{U}}(X) \times C^{\infty}\left(X \times U, \mathbb{R}^{p}\right) \mid \mathscr{P}_{1}(f, h) \text { is true }\right\}
$$


contains a residual set of $\operatorname{Diff}_{\mathrm{U}}(X) \times C^{\infty}\left(X \times U, \mathbb{R}^{p}\right)$. In Lemma 3, we will prove that, for a given $f \in \operatorname{Diff} \mathrm{U}(X)$, a given integer $r \geq 1$, and for every integer $n$, there exists a subset $U_{n}^{r}(f)$ of $C^{\infty}\left(X \times U, \mathbb{R}^{p}\right)$, open and dense for the $C^{r}$ topology, such that if $h$ belongs to the intersection $\bigcap_{n \geq 0} U_{n}^{r}(f)$, the pair $(f, h)$ satisfies property $\mathscr{P}_{2}$. Moreover, we will prove that, for every integer $n$, the set

$$
\mathscr{U}_{n}^{r}=\bigcup_{f \in \operatorname{Diff}_{\mathrm{U}}(X)}\{f\} \times U_{n}^{r}(f)
$$

is open dense in $\operatorname{Diff}_{\mathrm{U}}(X) \times C^{\infty}\left(X \times U, \mathbb{R}^{p}\right)$ equipped with the $C^{r}$ topology. Hence, clearly, the set $E_{1} \cap\left(\bigcap_{n \geq 0} \mathscr{U}_{n}^{r}\right)$ contains a residual set for the $C^{\infty}$ topology and a pair $(f, h)$ belonging to this set satisfies both properties $\mathscr{P}_{1}$ and $\mathscr{P}_{2}$. We will give two definitions before stating our lemmas.

Definition 4. Let $f \in \operatorname{Diff}_{\mathrm{U}}(X)$, we will say that the point $\left(x, \underline{u_{2 n+1}}\right) \in X \times U^{2 n+1}$ is periodic for $f$ if there exist two different integers $k$ and $k^{\prime}$ in $\{0, \ldots, 2 n\}$ such that $f^{k}\left(\overline{x, \underline{u_{k}}}\right)=f^{k^{\prime}}\left(x, \underline{u_{k^{\prime}}}\right)$.

Notations. We denote by $\mathscr{P}_{f}$ the set of all periodic points of $f$ and by $P_{f}$ the subset of $X^{(2)} \times U^{2 n+1}$ defined by:

$$
P_{f}=\left\{\left(x_{0}, \bar{x}_{0}, \underline{u_{2 n+1}}\right) \in X^{(2)} \times U^{2 n+1} \mid\left(x_{0}, \underline{u_{2 n+1}}\right) \text { and }\left(\bar{x}_{0}, \underline{u_{2 n+1}}\right) \text { periodic }\right\} .
$$

We denote by $P_{f}^{c}$ the set complement of $P_{f}$ in $X^{(2)} \times U^{2 n+1}$ :

$$
P_{f}^{c}=X^{(2)} \times U^{2 n+1} \backslash P_{f}
$$

We will divide $P_{f}^{c}$ into two parts.

Definition 5. We will say that the element $\left(x_{0}, \bar{x}_{0}, \underline{u_{2 n+1}}\right)$ of $P_{f}^{c}$ is permutable if there exist indices $\left(i_{1}, \ldots, i_{r}\right)$

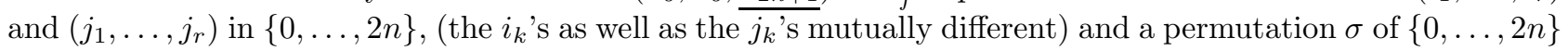
such that

and the equalities

$$
j_{k}=\sigma\left(i_{k}\right) \quad \text { for } k=1, \ldots, r
$$

are satisfied for all $k=1, \ldots, r$.

$$
\left(f^{i_{k}}\left(x_{0}, \underline{u_{i_{k}}}\right), u_{i_{k}}\right)=\left(f^{j_{k}}\left(\bar{x}_{0}, \underline{u_{j_{k}}}\right), u_{j_{k}}\right)
$$

Notice that in this definition, one cannot have $j_{k}=i_{k}$ because this would imply that $x_{0}=\bar{x}_{0}(f$ being a parameterized diffeomorphism).

Notations. A parameterized diffeomorphism $f$ being given, we denote by $\mathfrak{S}_{f}$ the subset of $P_{f}^{c}$ defined by

$$
\mathfrak{S}_{f}=\left\{\left(x_{0}, \bar{x}_{0}, \underline{u_{2 n+1}}\right) \in P_{f}^{c} \mid\left(x_{0}, \bar{x}_{0}, \underline{u_{2 n+1}}\right) \text { is permutable }\right\}
$$

and by $\mathfrak{S}_{f}^{c}$ the set complement of $\mathfrak{S}_{f}$ in $P_{f}^{c}$ :

$$
\mathfrak{S}_{f}^{c}=P_{f}^{c} \backslash \mathfrak{S}_{f}
$$

Clearly, for every $f$ in $\operatorname{Diff}_{U}(X)$, we have

$$
X^{(2)} \times U^{2 n+1}=P_{f} \cup \mathfrak{S}_{f} \cup \mathfrak{S}_{f}^{c}
$$

the union being disjoint. The proof of our result is based on the three following lemmas: 
Lemma 1. Let $A_{1}$ be the set of mappings $(f, h) \in \operatorname{Diff}_{\mathrm{U}}(X) \times C^{\infty}\left(X \times U, \mathbb{R}^{p}\right)$ such that:

$$
\Theta_{2 n+1}^{f, h}\left(x_{0}, \underline{u_{2 n+1}}\right) \neq \Theta_{2 n+1}^{f, h}\left(\bar{x}_{0}, \underline{u_{2 n+1}}\right) \quad \text { for all }\left(x_{0}, \bar{x}_{0}, \underline{u_{2 n+1}}\right) \in P_{f} .
$$

Set $A_{1}$ contains a residual subset $O_{1}$ of $\operatorname{Diff}_{U} \times C^{\infty}\left(X \times U, \mathbb{R}^{p}\right)$.

Lemma 2. Let $A_{2}$ be the set of mappings $(f, h) \in \operatorname{Diff}_{\mathrm{U}}(X) \times C^{\infty}\left(X \times U, \mathbb{R}^{p}\right)$ such that:

$$
\Theta_{2 n+1}^{f, h}\left(x_{0}, \underline{u_{2 n+1}}\right) \neq \Theta_{2 n+1}^{f, h}\left(\bar{x}_{0}, \underline{u_{2 n+1}}\right) \quad \text { for all }\left(x_{0}, \bar{x}_{0}, \underline{u_{2 n+1}}\right) \in \mathfrak{S}_{f} .
$$

Set $A_{2}$ contains a residual subset $O_{2}$ of $\operatorname{Diff}_{\mathrm{U}}(X) \times C^{\infty}\left(X \times U, \mathbb{R}^{p}\right)$.

In the third lemma $p$ denotes the first projection from $\operatorname{Diff}_{\mathrm{U}}(X) \times C^{\infty}\left(X \times U, \mathbb{R}^{p}\right)$ to $\operatorname{Diff}_{\mathrm{U}}$.

Lemma 3. Let $f$ be a given diffeomorphism in $\operatorname{Diff}_{\mathrm{U}}(X)$. There exists a sequence $\left(U_{n}(f)\right)_{n \geq 1}$ of open dense sets included in $C^{\infty}\left(X \times U, \mathbb{R}^{p}\right)$ such that for every mapping $h$ in $\bigcap_{n \geq 1} U_{n}(f)$, we have

$$
\Theta_{2 n+1}^{f, h}\left(x_{0}, \underline{u_{2 n+1}}\right) \neq \Theta_{2 n+1}^{f, h}\left(\bar{x}_{0}, \underline{u_{2 n+1}}\right) \text { for all }\left(x_{0}, \bar{x}_{0}, \underline{u_{2 n+1}}\right) \in \mathfrak{S}_{f}^{c} .
$$

Moreover for every integer $n$, the set

$$
\bigcup_{f \in \operatorname{Diff}_{\mathrm{U}}(X)}\{f\} \times U_{n}(f)
$$

is open dense in $\operatorname{Diff}_{\mathrm{U}}(X) \times C^{\infty}\left(X \times U, \mathbb{R}^{p}\right)$.

Properties $\mathscr{P}_{1}$ and $\mathscr{P}_{2}$. We say that the pair $(f, h) \in \operatorname{Diff}_{\mathrm{U}}(X) \times C^{\infty}\left(X \times U, \mathbb{R}^{p}\right)$ satisfies property $\mathscr{P}_{1}$ if it satisfies inequalities (4) and (5) and that it satisfies property $\mathscr{P}_{2}$ if inequality (6) is satisfied. Obviously, the injectivity of $\Theta_{2 n+1}^{f, h}$ is equivalent to $\mathscr{P}_{1}$ and $\mathscr{P}_{2}$ and so the proof of our main result reduces to proving these three lemmas.

\section{Proof of the main Result}

\subsection{Proof of Lemma 1}

The demonstration of this lemma is very technical and is based on the use of the multijet transversality theorem. We will introduce some new notations: $f \in$ Diff $_{\mathrm{U}}$ being given, for an index $s \in 1, \ldots, 2 n$ we denote by $\mathscr{P}_{f}^{s}$ the subset of elements $\left(x_{0}, \underline{u_{2 n+1}}\right) \in \mathscr{P}_{f}$ defined by the two conditions:

- $\forall i, j \in\{0, \ldots, s-1\}, f^{i}\left(x_{0}, \underline{u_{i}}\right) \neq f^{j}\left(x_{0}, u_{j}\right)$;

- $\exists s^{\prime} \in\{0, \ldots, s-1\} \mid f^{s^{\prime}}\left(x_{0}, \underline{u_{s^{\prime}}}\right)=f^{s}\left(x_{0}, \underline{u_{s}}\right)$.

Obviously, we have $\bigcup_{s=1}^{2 n} \mathscr{P}_{f}^{s}=\mathscr{P}_{f}$; since a finite intersection of open dense sets is an open dense set, we will prove lemma 1 for all elements $\left(x_{0}, \bar{x}_{0}, \underline{u_{2 n+1}}\right) \in P_{f}$ such that $\left(x_{0}, \underline{u_{2 n+1}}\right) \in \mathscr{P}_{f}^{s_{1}}$ and $\left(\bar{x}_{0}, \underline{u_{2 n+1}}\right) \in \mathscr{P}_{f}^{s_{2}}$, for every pair of elements $\left(s_{1}, s_{2}\right)$ in $\{1, \ldots, \overline{2 n\}}$.

Let $\left(x_{0}, \underline{u_{2 n+1}}\right) \in \mathscr{P}_{f}^{s_{1}}$ and $\left(\bar{x}_{0}, \underline{\bar{u}_{2 n+1}}\right) \in \mathscr{P}_{f}^{s_{2}}$, we can suppose without loss of generality that $s_{1} \geq s_{2}$. There

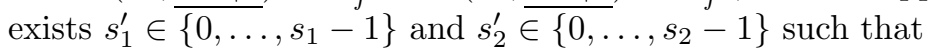

- $f^{s_{1}}\left(x_{0}, \underline{u_{s_{1}}}\right)=f^{s_{1}^{\prime}}\left(x_{0}, u_{s_{1}^{\prime}}\right)$ and $f^{s_{2}}\left(\bar{x}_{0}, \underline{\bar{u}_{s_{2}}}\right)=f^{s_{2}^{\prime}}\left(\bar{x}_{0}, \bar{u}_{s_{2}^{\prime}}\right)$;

- in addition $f^{i}\left(x_{0}, \underline{u_{i}}\right) \overline{\neq} f^{j}\left(x_{0}, \underline{u_{j}}\right)$ for all $i, j \in\left\{0, \ldots, s_{1}-1\right\}$ and $f^{i}\left(\bar{x}_{0}, \underline{u_{i}}\right) \neq f^{j}\left(\bar{x}_{0}, \underline{u_{j}}\right)$, for all $i, j \in\left\{0, \ldots, s_{2}-1\right\}$.

We let

$$
\begin{array}{rlrl}
x_{i} & =f^{i}\left(x_{0}, \underline{u_{i}}\right) & z_{i}=f\left(x_{i}, u_{i}\right) & y_{i}=h\left(x_{i}, u_{i}\right) \\
\bar{x}_{i}=f^{i}\left(\bar{x}_{0}, \underline{\bar{u}_{i}}\right) & z_{i}=f\left(\bar{x}_{i}, \bar{u}_{i}\right) & y_{i}=h\left(\bar{x}_{i}, \bar{u}_{i}\right) .
\end{array}
$$


In order to use multijet transversality theorem, we will study the equalities between the $x_{i}$ 's, $z_{i}$ 's, $y_{i}$ 's and the $\bar{x}_{i}$ 's, $\bar{z}_{i}$ 's, $\bar{y}_{i}$ 's. Consider the two following lists :

$$
\begin{array}{ll}
L_{1} & \left(x_{0}, u_{0}, z_{0}, y_{0}\right), \ldots,\left(x_{s_{1}-1}, u_{s_{1}-1}, z_{s_{1}-1}, y_{s_{1}-1}\right) \\
L_{2} & \left(\bar{x}_{0}, \bar{u}_{0}, \bar{z}_{0}, \bar{y}_{0}\right), \ldots,\left(\bar{x}_{s_{1}-1}, \bar{u}_{s_{1}-1}, \bar{z}_{s_{1}-1}, \bar{y}_{s_{1}-1}\right) .
\end{array}
$$

The elements of list $L_{1}$ are mutually distinct, but this is not necessarily true for the elements of list $L_{2}$, moreover it is possible that some elements of the first list are equal to some elements of the second one. Let us notice that two elements $\left(x_{i}, u_{i}, z_{i}, y_{i}\right)$ and $\left(\bar{x}_{j}, \bar{u}_{j}, \bar{z}_{j}, \bar{y}_{j}\right)$, (resp. $\left(\bar{x}_{i}, \bar{u}_{i}, \bar{z}_{i}, \bar{y}_{i}\right)$ and $\left.\left(\bar{x}_{j}, \bar{u}_{j}, \bar{z}_{j}, \bar{y}_{j}\right)\right)$ are equal if and only if $\left(x_{i}, u_{i}\right)$ is equal to $\left(\bar{x}_{j}, \bar{u}_{j}\right)$ (resp. $\left(\bar{x}_{i}, \bar{u}_{i}\right)$ is equal to $\left.\left(\bar{x}_{j}, \bar{u}_{j}\right)\right)$.

Hereafter, if $E$ is a finite set, card $E$ denotes the number of elements in $E$. Our strategy is the following: from the lists $L_{1}$ and $L_{2}$ we will show that it is possible to extract lists $\mathscr{L}_{1}$ and $\mathscr{L}_{2}$ such that

- the elements of the union $\mathscr{L}_{1} \cup \mathscr{L}_{2}$ are mutually distinct;

- there are card $\mathscr{L}_{1}+$ card $\mathscr{L}_{2}$ non redundant equalities between the elements $x_{i}$ 's, $z_{i}$ 's, $\bar{x}_{i}$ 's and $\bar{z}_{i}$ 's of these two lists; there are card $\mathscr{L}_{1}$ non redundant equalities between the $u_{i}$ 's and the $\bar{u}_{i}$ 's.

For each index $k$ such that $0 \leq k \leq s_{1}-1$, consider the set of indices

$$
I(k)=\left\{i \in\left\{0, \ldots, s_{1}-1\right\} \mid\left(x_{k}, u_{k}\right)=\left(\bar{x}_{i}, \bar{u}_{i}\right)\right\}
$$

We notice that the sets $I(k)$ are all disjoint (possibly empty) and that, under the assumptions $\underline{u_{2 n+1}}=\underline{\bar{u}_{2 n+1}}$ and $x_{0} \neq \bar{x}_{0}$, we have $k \notin I(k)$ because the equality $f^{k}\left(x_{0}, \underline{u_{k}}\right)=f^{k}\left(\bar{x}_{0}, \bar{u}_{k}\right)$ implies $x_{0}=\bar{x}_{0}$.

Definition 6. We will call division of $\left\{0,1, \ldots, s_{1}-1\right\}$ a sequence of $s_{1}$ subsets $I(0), \ldots, I\left(s_{1}-1\right)$ of $\left\{0, \ldots, s_{1}-1\right\}$ (possibly empty) mutually disjoint and such that $k \notin I(k)$ (for $0 \leq k \leq s_{1}-1$ ).

A division $\left(I(0), \ldots, I\left(s_{1}-1\right)\right)$ being given, we will say that the elements $\left(x_{0}, u_{2 n+1}\right)$ and $\left(\bar{x}_{0}, \bar{u}_{2 n+1}\right)$ in $\mathscr{P}_{f}^{s_{1}}$ and $\mathscr{P}_{f}^{s_{2}}\left(s_{1} \geq s_{2}\right)$ respectively, are in the configuration $\left(I(0), \ldots, I\left(s_{1}-1\right)\right)$ if we have $u_{k}=\bar{u}_{k}$ for $\overline{k=0}, \ldots, 2 n$ and if the set of indices $i$ such that $0 \leq i \leq s_{1}-1$ and $\left(x_{k}, u_{k}\right)=\left(\bar{x}_{i}, \bar{u}_{i}\right)$ is equal to $I(k)$ for $k=0, \ldots, s_{1}-1$.

Now let $\left(I(0), \ldots, I\left(s_{1}-1\right)\right)$ be a division of $\left\{0,1, \ldots, s_{1}-1\right\}$, let $\left(x_{0}, u_{2 n+1}\right)$ and $\left(\bar{x}_{0}, \bar{u}_{2 n+1}\right)$ be in $\mathscr{P}_{f}^{s_{1}}$ and $\mathscr{P}_{f}^{s_{2}}\left(s_{1} \geq s_{2}\right)$ respectively, in the configuration $\left(I(0), \ldots, I\left(s_{1}-1\right)\right)$. By writing all the equalities between the elements of list $L_{1}$ and the elements of list $L_{2}$ we can have equalities between the $u_{i}$ 's and the $\bar{u}_{j}$ 's. Under the assumption $\underline{u_{2 n+1}}=\bar{u}_{2 n+1}$, some equalities can be redundant; we will examine this possibility.

Definition 7. A division $\left(I(0), \ldots, I\left(s_{1}-1\right)\right)$ of $\left\{0, \ldots, s_{1}-1\right\}$ being given, we will say that the sequence $I\left(i_{1}\right), \ldots, I\left(i_{r}\right)$ is a chain if:

$$
i_{1} \in I\left(i_{2}\right), i_{2} \in I\left(i_{3}\right), \ldots, i_{r-1} \in I\left(i_{r}\right), i_{r} \in I\left(i_{1}\right)
$$

Notice that a chain is defined up to a circular permutation. We will see that two chains are disjoint or identical: let $I\left(i_{1}\right), \ldots, I\left(i_{r}\right)$ and $I\left(j_{1}\right), \ldots, I\left(j_{t}\right)$ be two chains with $r \leq t$. If these two chains are not disjoint, we can suppose that $I\left(i_{1}\right)=I\left(j_{1}\right)$ thus $i_{1}=j_{1}$ and consequently $i_{1} \in I\left(i_{2}\right) \cap I\left(j_{2}\right)$ which implies $i_{2}=j_{2}$. Reasoning by induction, we show the following equalities

$$
i_{1}=j_{1}, i_{2}=j_{2}, \ldots, i_{r}=j_{r}
$$

Now, we cannot have $r<t$ because this would imply $i_{r}=j_{r} \in I\left(i_{1}\right) \bigcap I\left(j_{r+1}\right)$ and so $i_{1}=j_{r+1}$ which leads to $j_{r+1}=j_{1}$ which is impossible.

Concerning the chains, we make another important remark. Let $I\left(i_{1}\right), \ldots, I\left(i_{r}\right)$ be a chain, by definition we can write the equalities

$$
u_{i_{2}}=\bar{u}_{i_{1}} \quad u_{i_{3}}=\bar{u}_{i_{2}} \quad \ldots \quad u_{i_{r}}=\bar{u}_{i_{r-1}} \quad u_{i_{1}}=\bar{u}_{i_{r}} \text {. }
$$


Under the assumption $\underline{u_{2 n+1}}=\underline{\bar{u}_{2 n+1}}$, we deduce from that

$$
u_{i_{2}}=u_{i_{1}} \quad u_{i_{3}}=u_{i_{2}} \quad \ldots \quad u_{i_{r}}=u_{i_{r-1}} \quad u_{i_{1}}=u_{i_{r}}
$$

but it is clear that the equality $u_{i_{1}}=u_{i_{r}}$ results from the $r-1$ first ones. Conversely, suppose that we can write the equalities

$$
u_{i_{1}}=u_{j_{1}} \quad \ldots \quad u_{i_{r}}=u_{j_{r}}
$$

with the $j_{k}$ 's all distinct and $j_{k} \in I\left(i_{k}\right)$ for $k=1, \ldots, r$, then if one equality can be deduced from the others, we can find a chain among the sets $I\left(i_{1}\right), \ldots, I\left(i_{r}\right)$. Suppose indeed that the equality $u_{i_{r}}=u_{j_{r}}$ can be deduced from the $r-1$ preceding equalities, then there exist two sequences $\left(i_{k_{1}}, \ldots, i_{k_{s}}\right)$ and $\left(j_{k_{1}}, \ldots, j_{k_{s}}\right)$ of elements of the sets $\left\{i_{1}, \ldots, i_{r}\right\}$ and $\left\{j_{1}, \ldots, j_{r}\right\}$ respectively such that

$$
i_{k_{1}}=j_{r} \quad i_{k_{2}}=j_{k_{1}} \quad \ldots \quad i_{k_{s}}=j_{k_{s-1}} \quad i_{r}=j_{k_{s}}
$$

and

$$
u_{i_{k_{1}}}=u_{j_{k_{1}}} \quad \ldots \quad u_{i_{k_{s}}}=u_{j_{k_{s}}} .
$$

Then we can write:

$$
i_{k_{1}}=j_{r} \in I\left(i_{r}\right) \quad i_{r}=j_{k_{s}} \in I\left(i_{k_{s}}\right) \quad i_{k_{s}}=j_{k_{s-1}} \in I\left(i_{k_{s-1}}\right) \quad \ldots \quad i_{k_{2}}=j_{k_{1}} \in I\left(i_{k_{1}}\right)
$$

which proves that $I\left(i_{k_{1}}\right), I\left(i_{r}\right), I\left(i_{k_{s}}\right), \ldots, I\left(i_{k_{2}}\right)$ is a chain.

Now we will count the number of non redundant equalities appearing between the elements of lists $L_{1}$ and $L_{2}$; in what follows $\ell$ will denote the number of chains in the sequence $\left(I(0), \ldots, I\left(s_{1}-1\right)\right)$ and we put

$$
q=s_{1}-\sum_{k=0}^{s_{1}-1} \operatorname{card} I(k) .
$$

In the following, we will consider two cases.

\subsubsection{Case where $\ell=0$}

We start by showing that, in this case, $q>0$; to do that, we will show that $q=0$ implies $\ell \neq 0$. Suppose that $q=0$, then we have $\bigcup_{k=0}^{s_{1}-1} I(k)=\left\{0, \ldots, s_{1}-1\right\}$. Let $i_{1}$ such that $I\left(i_{1}\right) \neq \varnothing$, as $i_{1} \notin I\left(i_{1}\right)$ there exists $i_{2} \neq i_{1}$ such as $i_{1} \in I\left(i_{2}\right)$, in the same way there exists $i_{3}$ such as $i_{2} \in I\left(i_{3}\right)$ and we can then write

$$
i_{1} \in I\left(i_{2}\right), i_{2} \in I\left(i_{3}\right), \ldots, i_{k} \in I\left(i_{k+1}\right), \ldots
$$

Now the sequence $\left(i_{k}\right)_{k \geq 1}$ is finite, so there exists $k<l$ such that $i_{k}=i_{l}$. Notice that $l \neq k+1$ (if not, we would have $\left.i_{l} \in I(l)\right)$, we can then write

$$
i_{k} \in I\left(i_{k+1}\right), i_{k+1} \in I\left(i_{k+2}\right), \ldots, i_{l-1} \in I(l)=I\left(i_{k}\right)
$$

which proves that $I(k), \ldots, I(l-1)$ is a chain and so $\ell \geq 1$.

Consider now the lists $L_{1}$ and the list $L_{2}^{\prime}$ extracted from $L_{2}$ by cancelling all the terms whose indices belong to the union of the $I(k)$ 's, let

$$
L_{2}^{\prime} \quad\left(\bar{x}_{r_{1}}, \bar{u}_{r_{1}}, \bar{z}_{r_{1}}, \bar{y}_{r_{1}}\right), \ldots,\left(\bar{x}_{r_{q}}, \bar{u}_{r_{q}}, \bar{z}_{r_{q}}, \bar{y}_{r_{q}}\right)
$$


with $r_{1}<r_{2}<\ldots<r_{q}$. In list $L_{2}^{\prime}$, there can exist equalities between some terms. In each equality class, we remove all terms but the one of highest index. We obtain then the list $L_{2}^{\prime \prime}$.

$$
L_{2}^{\prime \prime} \quad\left(\bar{x}_{t_{1}}, \bar{u}_{t_{1}}, \bar{z}_{t_{1}}, \bar{y}_{t_{1}}\right), \ldots,\left(\bar{x}_{t_{q^{\prime}}}, \bar{u}_{t_{q^{\prime}}}, \bar{z}_{t_{q^{\prime}}}, \bar{y}_{t_{q^{\prime}}}\right) \text {. }
$$

We will exhibit $s_{1}+q^{\prime}$ independent equalities between the $x_{i}$ 's, $z_{i}$ 's, $\bar{x}_{i}$ 's and $\bar{z}_{i}$ 's and $s_{1}$ independent equalities between the $u_{i}$ 's and $\bar{u}_{i}$ 's. First, we can write:

$$
z_{0}=x_{1}, z_{1}=x_{2}, \ldots, z_{s_{1}-1}=x_{s_{1}^{\prime}}
$$

which gives us $s_{1}$ equalities (the last one comes from $z_{s_{1}-1}=x_{s_{1}}$ and $x_{s_{1}}=x_{s_{1}^{\prime}}$ ).

We will now show that there are at least $q^{\prime}$ equalities between the terms of $L_{2}^{\prime \prime}$ and between the terms $x_{j}$ and $\bar{x}_{j}$. Let us examine two consecutive terms in $L_{2}^{\prime \prime}:\left(\bar{x}_{t_{i}}, \bar{u}_{t_{i}}, \bar{z}_{t_{i}}, \bar{y}_{t_{i}}\right),\left(\bar{x}_{t_{i+1}}, \bar{u}_{t_{i+1}}, \bar{z}_{t_{i+1}}, \bar{y}_{t_{i+1}}\right)$ with $i \in\left\{1, \ldots, q^{\prime}-1\right\}$.

- Suppose that $t_{i+1}=t_{i}+1$, we have in this case $\bar{z}_{t_{i}}=\bar{x}_{t_{i+1}}$;

- if $t_{i+1}>t_{i}+1$, the term $\left(\bar{x}_{t_{i}+1}, \bar{u}_{t_{i}+1}, \bar{z}_{t_{i}+1}, \bar{y}_{t_{i}+1}\right)$ was removed because

- it is equal to a term of $L_{1}$ and consequently there exists $j \in\left\{0, \ldots, s_{1}-1\right\}$ with $j \neq t_{i}+1$ and $\bar{x}_{t_{i}+1}=x_{j}$, from what it follows $\bar{z}_{t_{i}}=x_{j}$;

- or it is equal to a term of list $L_{2}^{\prime \prime}$ and consequently there exists $j \in\left\{i+1, \ldots, q^{\prime}\right\}$ such that $t_{j}>t_{i}+1$ and $\bar{x}_{t_{i}+1}=\bar{x}_{t_{j}}$, so $\bar{z}_{t_{i}}=\bar{x}_{t_{j}}$ with $t_{j}>t_{i}$.

At this point we have obtained $s_{1}+q^{\prime}-1$ equalities, in the following, we distinguish two situations. We start by examining the case where $t_{q^{\prime}}<s_{1}-1$ : in this case, the term $\left(\bar{x}_{t_{q^{\prime}}+1}, \bar{u}_{\left.t_{q^{\prime}+1}, \bar{x}_{t_{q^{\prime}}+2}, \bar{y}_{t_{q^{\prime}}+1}\right) \text { was removed }}\right.$ because it is equal to a term of $L_{1}$, hence there exists $j \in\left\{1, \ldots, s_{1}-1\right\}$ with $j \neq t_{q^{\prime}}+1$ and $\bar{x}_{t_{q^{\prime}}+1}=x_{j}$ and so $\bar{z}_{t_{q^{\prime}}}=x_{j}$, which gives us an additional equality.

The second situation occurs when $t_{q^{\prime}}=s_{1}-1$, and it is subdivided into two cases

- if $s_{1}=1$ or if $\left(\bar{x}_{j}, \bar{u}_{j}, \bar{z}_{j}, \bar{y}_{j}\right) \in L_{2}^{\prime \prime}$ for $j=0, \ldots, s_{1}-2$, list $L_{2}^{\prime \prime}$ has $s_{1}$ terms and, since $s_{1} \geq s_{2}$, we have the equality $\bar{x}_{s_{2}}=\bar{x}_{s_{2}^{\prime}}$;

- if $s_{1} \geq 2$ and if there exists $0 \leq j \leq s_{1}-2$ such that $\left(\bar{x}_{j}, \bar{u}_{j}, \bar{z}_{j}, \bar{y}_{j}\right) \notin L_{2}^{\prime \prime}$, we put

$$
r=\max \left\{j \in\left\{0, \ldots, s_{1}-2\right\} \mid\left(\bar{x}_{j}, \bar{u}_{j}, \bar{z}_{j}, \bar{y}_{j}\right) \notin L_{2}^{\prime \prime}\right\} .
$$

Now, the term $\left(\bar{x}_{r}, \bar{u}_{r}, \bar{z}_{r}, \bar{y}_{r}\right)$ was removed because

- it is equal to a term of list $L_{1}$, hence there exists $j \in\left\{0, \ldots, s_{1}-1\right\}$ (with $j \neq r$ ) such that $\bar{z}_{r}=z_{j}$ and so $\bar{x}_{r+1}=z_{j}$ which is an additional equality;

- or it is equal to a term of list $L_{2}^{\prime}$, hence there exists $t_{i}$ such that $r<t_{i} \leq s_{1}-1$ and $\bar{z}_{r}=\bar{z}_{t_{i}}$, and so $\bar{x}_{r+1}=\bar{z}_{t_{i}}$ with $r+1 \leq t_{i}$ which is an additional equality.

At this point of our reasoning, we can conclude to the existence of $s_{1}+q^{\prime}$ equalities between the terms $x_{i}$ ', $z_{i}$, $\bar{x}_{i}$ and $\bar{z}_{i}$ in lists $L_{1}$ and $L_{2}^{\prime \prime}$; we examine now the relation between the $u_{i}$ 's and the $\bar{u}_{i}$ 's.

For a given $k$ such that $I(k)$ is nonempty we let $I(k)=\left\{l_{1}, \ldots, l_{\alpha}\right\}$ and we can write the equalities

$$
u_{k}=\bar{u}_{l_{1}}, \ldots, u_{k}=\bar{u}_{l_{\alpha}}
$$

Under the assumption $\underline{u_{2 n+1}}=\underline{\bar{u}_{2 n+1}}$, we deduce

$$
u_{k}=u_{l_{1}}, \ldots, u_{k}=u_{l_{\alpha}} .
$$

which are $\alpha$ equalities between the $u_{i}$ 's. Repeating the reasoning for each $I(k)$ we get

$$
\sum_{k=0}^{s_{1}-1} \operatorname{card} I(k)=s_{1}-q
$$

equalities between the $u_{i}$ 's (since there is no chain, there is no redundant equalities). 
Let us examine now the list $L_{2}^{\prime}$. We denote by $C_{1}, \ldots, C_{q^{\prime}}$ the classes of equalities; recall that, for the construction of list $L_{2}^{\prime \prime}$, we kept the term of higher index in each class. For each index $t_{i}$, we can write card $C_{i}-1$ equalities between $\bar{u}_{t_{i}}$ and terms $\bar{u}_{j}$ with $j<t_{i}$ and $j \notin\left\{t_{1}, \ldots, t_{q^{\prime}}\right\}$, under the hypothesis $\underline{u}_{2 n}=\underline{\bar{u}_{2 n}}$, we deduce card $C_{i}-1$ equalities between $\bar{u}_{t_{i}}$ and terms $u_{j}$ with $j<t_{i}$, we can write also the $q^{\prime}$ equalities

$$
\bar{u}_{t_{1}}=u_{t_{1}}, \ldots, \bar{u}_{t_{q}^{\prime}}=u_{t_{q}^{\prime}}
$$

therefore, we have an amount of

$$
\sum_{i=1}^{q^{\prime}}\left(\operatorname{card} C_{i}-1\right)+q^{\prime}=q
$$

equalities between the $\bar{u}_{i}$ 's and the $u_{i}$ 's in lists $L_{1}$ and $L_{2}^{\prime \prime}$.

Conclusion. In this subsection, we have proved the existence of $s_{1}+q^{\prime}$ equalities between the $x_{i}$ 's, $z_{i}$ 's, $\bar{x}_{i}$ 's and $\bar{z}_{i}$ 's in lists $L_{1}$ and $L_{2}^{\prime \prime}$ and $s_{1}$ equalities between the $u_{i}$ 's and the $\bar{u}_{i}$ 's.

\subsubsection{Case where $\ell \neq 0$}

In this case there exist $\ell$ chains denoted by

$$
\begin{array}{cc}
\mathscr{C}_{1} & I\left(i_{1}^{1}\right), \ldots, I\left(i_{n_{1}}^{1}\right) \\
\vdots \\
\mathscr{C}_{\ell} & I\left(i_{1}^{\ell}\right), \ldots, I\left(i_{n_{\ell}}^{\ell}\right)
\end{array}
$$

a chain being defined up to a circular permutation, we can suppose that, for $k=1, \ldots, \ell, i_{1}^{k} \neq 0$.

We built the list $L_{1}^{\prime}$ extracted from $L_{1}$ by removing the elements of indices $i_{1}^{1}, \ldots, i_{1}^{\ell}$. We consider also the list $L_{2}^{\prime \prime \prime}$ extracted from $L_{2}$ by taking the terms of the list $L_{2}^{\prime \prime}$ (possibly empty) which is obtained starting from $L_{2}$ as explained in the case $\ell=0$ and by adding the terms of indices $i_{n_{1}}^{1}, \ldots, i_{n_{\ell}}^{\ell}$. Notice that, due to the construction of $L_{2}^{\prime \prime}$, the lists $\left\{i_{n_{1}}^{1}, \ldots, i_{n_{\ell}}^{\ell}\right\}$ and $\left\{t_{1}, \ldots, t_{q^{\prime}}\right\}$ are disjoint, thus, the number of elements in $L_{2}^{\prime \prime \prime}$ is equal to $\ell+q^{\prime}$; we introduce the following notations:

$$
\begin{aligned}
\left\{i_{1}, \ldots, i_{s_{1}-\ell}\right\} & =\left\{0, \ldots, s_{1}-1\right\} \backslash\left\{i_{1}^{1}, \ldots, i_{1}^{\ell}\right\} & & \text { with } i_{1}<\ldots<i_{s_{1}-\ell} \\
\left\{j_{1}, \ldots, j_{\ell+q^{\prime}}\right\} & =\left\{i_{n_{1}}^{1}, \ldots, i_{n_{\ell}}^{\ell}, t_{1}, \ldots, t_{q^{\prime}}\right\} & & \text { with } j_{1}<\ldots<j_{\ell+q^{\prime}} .
\end{aligned}
$$

Notice that $i_{1}$ is necessarily zero and that, with these notations, lists $L_{1}^{\prime}$ and $L_{2}^{\prime \prime \prime}$ can be written:

$$
\begin{array}{ll}
L_{1}^{\prime} & \left(x_{i_{1}}, u_{i_{1}}, z_{i_{1}}, y_{i_{1}}\right), \ldots,\left(x_{i_{s_{1}-\ell}}, u_{i_{s_{1}-\ell}}, z_{i_{s_{1}-\ell}}, y_{i_{s_{1}-\ell}}\right) \\
L_{2}^{\prime \prime \prime} & \left(\bar{x}_{j_{1}}, \bar{u}_{j_{1}}, \bar{z}_{j_{1}}, \bar{y}_{j_{1}}\right), \ldots,\left(\bar{x}_{j_{\ell+q^{\prime}}}, \bar{u}_{j_{\ell+q^{\prime}}}, \bar{z}_{j_{\ell+q^{\prime}}}, \bar{y}_{j_{\ell+q^{\prime}}}\right) .
\end{array}
$$

It can be easily seen that the terms of $L_{1}^{\prime} \cup L_{2}^{\prime \prime \prime}$ are mutually distinct.

In the following, for the sake of readability, we will sometimes write $\tau(i)$ in place of $\tau_{i}$ where $\tau$ is one of the symbols $x, z, \bar{x}, \ldots$ and $i$ is an expression representing an index.

We start by showing that we have at least $s_{1}+q^{\prime}$ equalities between the terms $x_{i}, z_{i}, \bar{x}_{i}$ and $\bar{z}_{i}$ of the lists $L_{1}^{\prime}$ and $L_{2}^{\prime \prime \prime}$. We put $i_{s_{1}-\ell+1}=s_{1}$ and we examine first the terms corresponding to two consecutive indices $i_{r}$ and $i_{r+1}$ with $r \in\left\{1, \ldots, s_{1}-\ell-1\right\}$.

- if $i_{r+1}=i_{r}+1<s_{1}$, we can write the equality

$$
z\left(i_{r}\right)=x\left(i_{r+1}\right) ;
$$

if $i_{r+1}=i_{r}+1=s_{1}$, we have $x\left(s_{1}\right)=x\left(s_{1}^{\prime}\right)$ if $s_{1}^{\prime} \notin\left\{i_{1}^{1}, \ldots, i_{1}^{\ell}\right\}$, we deduce the equality

$$
z\left(i_{r}\right)=x\left(s_{1}^{\prime}\right)
$$


between two terms of $L_{1}^{\prime}$, if there exists $1 \leq a \leq \ell$ such that $s_{1}^{\prime}=i_{1}^{a}$, we have $x\left(i_{1}^{a}\right)=\bar{x}\left(i_{n_{a}}^{a}\right)$ and we can write the equality:

$$
z\left(i_{r}\right)=\bar{x}\left(i_{n_{a}}^{a}\right)
$$

- if $i_{r+1}=i_{r}+d$ with $2 \leq d \leq i\left(s_{1}-\ell\right)-i_{r}$, the index $i_{r}+1$ is equal to an index $i_{1}^{k_{1}}$, since $i_{n_{k_{1}}}^{k_{1}} \in I\left(i_{1}^{k_{1}}\right)$, we have $x\left(i_{1}^{k_{1}}\right)=\bar{x}\left(i_{n_{k_{1}}}^{k_{1}}\right)$, now $x\left(i_{1}^{k_{1}}\right)=x\left(i_{r}+1\right)=z\left(i_{r}\right)$, so we have the equality:

$$
z\left(i_{r}\right)=\bar{x}\left(i_{n_{k_{1}}}^{k_{1}}\right) .
$$

From the definition of $L_{1}^{\prime}$, it follows that for each $j=1, \ldots, d-1$, the index $i_{r+j}$ belongs to the list $\left(i_{1}^{1}, \ldots, i_{1}^{\ell}\right)$, so there exists $k_{j}$ such that $i_{r+j}=i_{1}^{k_{j}}$. If $i_{n_{k_{j}}}^{k_{j}}+1$ is the index of an element in the list $L_{2}^{\prime \prime \prime}$, we have the equality

$$
\bar{z}\left(i_{n_{k_{j}}}^{k_{j}}\right)=\bar{x}\left(i_{n_{k_{j}}}^{k_{j}}+1\right) .
$$

Otherwise, the term of index $i_{n_{k_{j}}}^{k_{j}}+1$ was canceled because it is equal to

- a term of $L_{1}^{\prime}$, which implies the existence of an index $i_{a}$ such that $\bar{x}\left(i_{n_{k_{j}}}^{k_{j}}+1\right)=x\left(i_{a}\right)$ and since $\bar{x}\left(i_{n_{k_{j}}}^{k_{j}}+1\right)=\bar{z}\left(i_{n_{k_{j}}}^{k_{j}}\right)$, we have

$$
\bar{z}\left(i_{n_{k_{j}}}^{k_{j}}\right)=x\left(i_{a}\right)
$$

- a term of $L_{1} \backslash L_{1}^{\prime}$, which implies the existence of an index $i_{1}^{b}$ such that $\bar{x}\left(i_{n_{k_{j}}}^{k_{j}}+1\right)=x\left(i_{1}^{b}\right)$ and since $\bar{x}\left(i_{n_{k_{j}}}^{k_{j}}+1\right)=\bar{z}\left(i_{n_{k_{j}}}^{k_{j}}\right)$ and $x\left(i_{1}^{b}\right)=\bar{x}\left(i_{n_{b}}^{b}\right)$, we can write the equality:

$$
\bar{z}\left(i_{n_{k_{j}}}^{k_{j}}\right)=\bar{x}\left(i_{n_{b}}^{b}\right)
$$

- a term of list $L_{2}^{\prime \prime}$, which implies the existence of an index $t_{i}$ such that $\bar{x}\left(i_{n_{k_{j}}}^{k_{j}}+1\right)=\bar{x}\left(t_{i}\right)$ and we can write:

$$
\bar{z}\left(i_{n_{k_{j}}}^{k_{j}}\right)=\bar{x}\left(t_{i}\right)
$$

At this point, we have written

$$
\sum_{r=1}^{s_{1}-\ell}\left(i_{r+1}-i_{r}\right)=i_{s_{1}-\ell+1}-i_{1}=i_{s_{1}-\ell+1}=s_{1}
$$

equalities between the $x_{i}$ 's, $z_{i}$ 's, $\bar{x}_{i}$ 's and $\bar{z}_{i}$ 's of lists $L_{1}^{\prime}$ and $L_{2}^{\prime \prime \prime}$.

Reasoning as in the case where $\ell=0$, the $q^{\prime}$ terms of the list $L_{2}^{\prime \prime}$ give $q^{\prime}$ equalities; notice that, since the sets of indices $\left\{i_{n_{1}}^{1}, \ldots, i_{n_{\ell}}^{\ell}\right\}$ and $\left\{t_{1}, \ldots, t_{q^{\prime}}\right\}$ are disjoint, these $q^{\prime}$ equalities are independent from the $s_{1}$ equalities written above.

We will now prove that we can write $s_{1}-\ell$ equalities between the terms $u_{i}$ and $\bar{u}_{j}$ in lists $L_{1}^{\prime}$ and $L_{2}^{\prime \prime \prime}$. Consider the chain $\mathscr{C}_{1}$ and let

$$
\begin{aligned}
I\left(i_{1}^{1}\right) & =\left\{i_{n_{1}}^{1}, i_{1,2}^{1}, \ldots, i_{1, m_{1}}^{1}\right\} \\
I\left(i_{2}^{1}\right) & =\left\{i_{1}^{1}, i_{2,2}^{1}, \ldots, i_{2, m_{2}}^{1}\right\} \\
& \vdots \\
I\left(i_{n_{1}}^{1}\right) & =\left\{i_{n_{1}-1}^{1}, i_{n_{1}, 2}^{1}, \ldots, i_{n_{1}, m_{n_{1}}}^{1}\right\} .
\end{aligned}
$$


We can write the following equalities

$$
\begin{array}{cccc}
u\left(i_{1}^{1}\right)=\bar{u}\left(i_{n_{1}}^{1}\right) & u\left(i_{1}^{1}\right)=\bar{u}\left(i_{1,2}^{1}\right) & \ldots & u\left(i_{1}^{1}\right)=\bar{u}\left(i_{1, m_{1}}^{1}\right) \\
\vdots & \vdots & \ldots & \vdots \\
u\left(i_{n_{1}}^{1}\right)=\bar{u}\left(i_{n_{1}-1}^{1}\right) & u\left(i_{n_{1}}^{1}\right)=\bar{u}\left(i_{n_{1}, 2}^{1}\right) & \ldots & u\left(i_{n_{1}}^{1}\right)=\bar{u}\left(i_{n_{1}, m_{n_{1}}}^{1}\right) .
\end{array}
$$

Taking into account that the term $u\left(i_{1}^{1}\right)$ does not appear in list $L_{1}^{\prime}$ and under the assumption that $\underline{u_{2 n+1}}=\underline{\bar{u}_{2 n+1}}$, we deduce the following equalities:

$$
\begin{array}{cccc}
u\left(i_{2}^{1}\right)=u\left(i_{2,2}^{1}\right) & \ldots & u\left(i_{2}^{1}\right)=u\left(i_{2, m_{2}}^{1}\right) \\
u\left(i_{3}^{1}\right)=u\left(i_{2}^{1}\right) & u\left(i_{3}^{1}\right)=u\left(i_{3,2}^{1}\right) & \ldots & u\left(i_{3}^{1}\right)=u\left(i_{3, m_{3}}^{1}\right) \\
\vdots & \vdots & \ldots & \vdots \\
u\left(i_{n_{1}}^{1}\right)=u\left(i_{n_{1}-1}^{1}\right) & u\left(i_{n_{1}}^{1}\right)=u\left(i_{n_{1}, 2}^{1}\right) & \ldots & u\left(i_{n_{1}}^{1}\right)=u\left(i_{n_{1}, m_{n_{1}}}^{1}\right) .
\end{array}
$$

So we have an amount of

$$
\sum_{j=1}^{n_{1}} \operatorname{card} I\left(i_{j}^{1}\right)-m_{1}-1
$$

equalities. From the first line of equalities (7) and under the assumption $\underline{u_{2 n+1}}=\underline{\bar{u}_{2 n+1}}$, we can write the $m_{1}-1$ equalities:

$$
\bar{u}\left(i_{n_{1}}^{1}\right)=u\left(i_{1,2}^{1}\right) \quad \bar{u}\left(i_{n_{1}}^{1}\right)=u\left(i_{1,3}^{1}\right) \quad \ldots \quad \bar{u}\left(i_{n_{1}}^{1}\right)=u\left(i_{1, m_{1}}^{1}\right) .
$$

Reasoning in the same way for the other chains, we obtain

$$
\sum_{i=1}^{\ell} \sum_{j=1}^{n_{i}} \operatorname{card} I\left(i_{j}^{i}\right)-2 \ell
$$

equalities between the $u_{i}$ 's and the $\bar{u}\left(i_{n_{j}}^{j}\right)$ 's. Taking into account the sets $I(k)$ which are not components of chains and reasoning as in the case $\ell=0$, we can write other equalities between the $u_{i}$ 's; these equalities added to the equalities above give us $\mathrm{t}$ an amount of

$$
s_{1}-q-2 \ell
$$

equalities between the $u_{i}$ 's and the $\bar{u}\left(i_{n_{j}}^{j}\right)$ 's; clearly, these equalities are non redundant because, due to the absence of the terms of indices $i_{1}^{1}, \ldots, i_{1}^{\ell}$ from $L_{1}^{\prime}$, we cannot find a chain built with indices appearing in list $L_{1}^{\prime}$.

Now reasoning as in the case $\ell=0$, we can write $q-q^{\prime}$ equalities between the $\bar{u}\left(t_{i}\right)$ 's and some $\bar{u}_{j}$ (with $j<t_{i}$ and $j \notin\left\{t_{1}, \ldots, t_{q^{\prime}}\right\}$ ), also we can write the following $q^{\prime}+\ell$ equalities:

$$
\begin{array}{lll}
\bar{u}\left(i_{t_{1}}\right)=u\left(i_{t_{1}}\right) & \ldots & \bar{u}\left(i_{t_{q^{\prime}}}\right)=u\left(i_{t_{q^{\prime}}}\right) \\
\bar{u}\left(i_{n_{1}}^{1}\right)=u\left(i_{n_{1}}^{1}\right) & \ldots & \bar{u}\left(i_{n_{\ell}}^{\ell}\right)=u\left(i_{n_{\ell}}^{\ell}\right) .
\end{array}
$$

Finally, we have an amount of $s_{1}-\ell$ equalities between the $u_{i}$ 's and the $\bar{u}_{j}$ 's and $s_{1}+q^{\prime}$ equalities between the $x_{i}$ 's, $z_{i}$ 's, $\bar{x}_{i}$ 's and $\bar{z}_{i}$ 's.

We are now ready to apply the multijet transversality theorem. For given $s_{1}$ and $s_{2}$, consider the set $(X \times U)^{\left(d_{1}+d_{2}\right)}$ with $1 \leq d_{1} \leq s_{1}$ and $d_{2} \leq d_{1}$, let us denote by $\alpha(=(x, u))$ an element of $X \times U$ and, for $(f, h)$ 
in $\operatorname{Diff}_{\mathrm{U}}(X) \times C^{\infty}\left(X \times U, \mathbb{R}^{p}\right)$, consider the mapping:

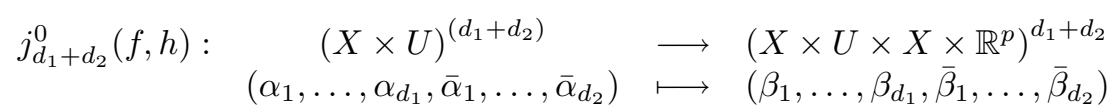

where $\beta_{i}=\left(x_{i}, u_{i}, f\left(x_{i}, u_{i}\right), h\left(x_{i}, u_{i}\right)\right)$ if $\alpha_{i}=\left(x_{i}, u_{i}\right)$ (analogous expression for the $\bar{\beta}_{i}$ 's). In the manifold $\left(X \times U \times X \times \mathbb{R}^{p}\right)^{d_{1}+d_{2}}$, we consider a submanifold $W$ defined by $d_{1}+d_{2}$ equalities between the $x_{i}$ 's, $z_{i}$ 's, $\bar{x}_{i}$ 's and $\bar{z}_{i}$ 's, $d_{1}$ equalities between the $u_{i}$ 's and the $\bar{u}_{i}$ 's and $d_{2}$ equalities between the elements $\bar{y}_{1}, \ldots, \bar{y}_{d_{2}}$ and $d_{2}$ elements chosen among the elements $y_{1}, \ldots, y_{d_{1}}$. The number of submanifolds such that $W$ is finite, moreover these submanifolds are closed and their codimensions are equal to

$$
\left(d_{1}+d_{2}\right) n+d_{1} m+d_{2} p
$$

which is greater than the dimension of $(X \times U)^{\left(d_{1}+d_{2}\right)}$, therefore transversality to $W$ means non membership and we can assert that the set of mappings $(f, h)$ belonging to $\operatorname{Diff}_{U}(X) \times C^{\infty}\left(X \times U, \mathbb{R}^{p}\right)$ such that

$$
j_{d_{1}+d_{2}}^{0}(f, h)\left(\alpha_{1}, \ldots, \alpha_{d_{1}}, \bar{\alpha}_{1}, \ldots, \bar{\alpha}_{d_{2}}\right) \notin W
$$

is residual. Now denote by $O_{s_{1}, s_{2}}$ the residual set in $\operatorname{Diff}_{\mathrm{U}} \times C^{\infty}\left(X \times U, \mathbb{R}^{p}\right)$ obtained as the finite intersection of all residual sets related to all possible values for $d_{1}$ and $d_{2}$ and all submanifolds such that $W$. Let $(f, h)$ in $O_{s_{1}, s_{2}}$ and assume that $\left(x_{0}, u_{2 n+1}\right) \in \mathscr{P}_{f}^{s_{1}}$ and $\left(\bar{x}_{0}, \bar{u}_{2 n+1}\right) \in \mathscr{P}_{f}^{s_{2}}$. If $u_{2 n+1}=\bar{u}_{2 n+1}$, as we have seen above, we can extract two lists $\mathscr{L}_{1}$ and $\overline{\mathscr{L}_{2}}$ from $L_{1}$ and $L_{2}$ of length $d_{1}$ and $d_{2}$ respectively such that:

- there exist $d_{1}+d_{2}$ equalities between the $x_{i}$ 's, $z_{i}$ 's, $\bar{x}_{i}$ 's and $\bar{z}_{i}$ 's;

- there exist $d_{1}$ equalities between the $u_{i}$ 's and the $\bar{u}_{i}$ 's.

If, in addition we suppose that the $d_{2}$ elements $\bar{y}_{i}$ in list $\mathscr{L}_{2}$ are equal to the corresponding $y_{i}$ in list $\mathscr{L}_{1}$, the element

$$
j_{f, h}^{0}\left(\alpha_{1}, \ldots, \alpha_{d_{1}}, \bar{\alpha}_{1}, \ldots, \bar{\alpha}_{d_{2}}\right)
$$

belongs to a submanifold such as $W$ (here $\alpha_{1}, \ldots, \alpha_{d_{1}}$ (resp. $\bar{\alpha}_{1}, \ldots, \bar{\alpha}_{d_{2}}$ ) denotes the list constituted by the projection of the elements of $\mathscr{L}_{1}$ (resp. $\mathscr{L}_{2}$ ) onto $X \times U$ ). Such a membership being impossible for a pair $(f, h)$ in $O_{s_{1}, s_{2}}$, there must exist a term $y_{i}$ different from $\bar{y}_{i}$. Finally we see that, denoting by $A_{1}$ the finite intersection of all residual sets $O_{s_{1}, s_{2}}$, Lemma 1 is proved.

\subsection{Proof of Lemma 2}

The demonstration of this lemma is very similar to the one of Lemma 1.

Let $\left(x_{0}, \bar{x}_{0}, \underline{u_{2 n+1}}\right)$ be in $\mathfrak{S}_{f}$ and suppose that $\left(x_{0}, \underline{u_{2 n+1}}\right) \notin \mathscr{P}_{f}$. There exist indices $i_{1}<\cdots<i_{r}$ and $j_{1}, \ldots, j_{r} \in\{0, \overline{\ldots, 2} n\}$ mutually distinct and a permutation $\sigma$ such that $j_{k}=\sigma\left(i_{k}\right)$ for $k=1, \ldots, r$ and

$$
\left(f^{i_{k}}\left(x_{0}, \underline{u_{i_{k}}}\right), u_{i_{k}}\right)=\left(f^{j_{k}}\left(\bar{x}_{0}, \underline{u_{j_{k}}}\right), u_{j_{k}}\right) \quad \text { for } k=1, \ldots, r .
$$

Given a finite sequence $\bar{u}_{2 n+1}$, with the same notations than in the proof of Lemma 1, consider the two following lists constituted by the terms of indices $i_{1}, i_{1}+1, \ldots, i_{r-1}-1, i_{r-1}$

$$
\begin{aligned}
& L_{1}:\left(x_{i_{1}}, u_{i_{1}}, z_{i_{1}}, y_{i_{1}}\right), \ldots,\left(x_{i_{r}-1}, u_{i_{r}-1}, z_{i_{r}-1}, y_{i_{r}-1}\right) \\
& L_{2}:\left(\bar{x}_{i_{1}}, \bar{u}_{i_{1}}, \bar{z}_{i_{1}}, \bar{y}_{i_{1}}\right), \ldots,\left(\bar{x}_{i_{r}-1}, \bar{u}_{i_{r}-1}, \bar{z}_{i_{r}-1}, \bar{y}_{i_{r}-1}\right) .
\end{aligned}
$$

Like in the proof of Lemma 1 , we will extract from them two lists $\mathscr{L}_{1}$ and $\mathscr{L}_{2}$ such that, under the assumption $\underline{u_{2 n+1}}=\underline{\bar{u}_{2 n+1}}$, 
- the elements of the union $\mathscr{L}_{1} \cup \mathscr{L}_{2}$ are all distinct;

- there are card $\mathscr{L}_{1}+$ card $\mathscr{L}_{2}$ non redundant equalities between the elements $x_{i}$ 's, $z_{i}$ 's, $\bar{x}_{i}$ 's and $\bar{z}_{i}$ 's of these two lists; there are card $\mathscr{L}_{1}$ non redundant equalities between the $u_{i}$ 's and the $\bar{u}_{i}$ 's.

From the definition of $\mathfrak{S}_{f}$, we can suppose, without loss of generality, that $\left(x_{0}, u_{2 n+1}\right) \notin \mathscr{P}_{f}$, so the elements of list $L_{1}$ are all distinct but this is not necessarily the case for the elements of list $L_{2}$. Moreover, it can happen that some elements of the first list are equal to elements of the second one.

Like in the demonstration of Lemma 1 , for each index $k\left(i_{1} \leq k \leq i_{r}-1\right)$, we consider the sets

$$
I(k)=\left\{i \mid i_{1} \leq i \leq i_{r}-1 \text { and }\left(x_{k}, u_{k}\right)=\left(\bar{x}_{i}, \bar{u}_{i}\right)\right\}
$$

which obviously have the same properties than in the proof of Lemma 1 . We introduce also the concepts of division and chains as in the demonstration of Lemma 1 . We denote by $\ell$ the number of chains and by $q$ the number

$$
q=i_{r}-i_{1}-\sum_{k=i_{1}}^{i_{r}-1} \operatorname{card} I(k) .
$$

Notice that $j_{r} \in\left\{i_{1}, i_{1}+1, \ldots, i_{r-1}\right\}$ (the set of all integers between $i_{1}$ and $i_{r}-1$ ) because we cannot have $j_{r}=i_{r}$, which would imply $x_{0}=\bar{x}_{0}$; moreover $\left(\bar{x}_{j_{r}}, \bar{u}_{j_{r}}, \bar{z}_{j_{r}}, \bar{y}_{j_{r}}\right)$ is different from all the elements of list $L_{1}$, indeed an equality such that $\bar{x}_{j_{r}}=x_{k}$ with $i_{1} \leq k \leq i_{r}-1$ would imply $x_{i_{r}}=x_{k}$ which is in contradiction with the fact that $\left(x_{0}, u_{2 n+1}\right) \notin \mathscr{P}_{f}$.

Consider the list $L_{2}^{\prime}$ extracted from $L_{2}$ by removing every term whose index belongs to the union of the $I_{k}$ 's; notice that the term of index $j_{r}$ is present in list $L_{2}^{\prime}$. In this list, there can exist equalities between some terms, in each equality class, we remove all terms but the one of highest index excepted for the equality class which contains the term of index $j_{r}$, for this class, we keep the term $\left(\bar{x}_{j_{r}}, \bar{u}_{j_{r}}, \bar{z}_{j_{r}}, \bar{y}_{j_{r}}\right)$. In this way, we obtain a list denoted by $L_{2}^{\prime \prime}$ :

$$
L_{2}^{\prime \prime}:\left(\bar{x}_{t_{1}}, \bar{u}_{t_{1}}, \bar{z}_{t_{1}}, \bar{y}_{t_{1}}\right), \ldots,\left(\bar{x}_{t_{q^{\prime}}}, \bar{u}_{t_{q^{\prime}}}, \bar{z}_{t_{q^{\prime}}}, \bar{y}_{t_{q^{\prime}}}\right) \quad \text { with } t_{1}<\cdots<t_{q^{\prime}}
$$

In what follows, we will distinguish two cases.

\subsubsection{Case where $\ell=0$}

In the first list we find the $i_{r}-i_{1}-1$ following equalities

$$
z_{k}=x_{k+1} \quad \text { for } k=i_{1}, i_{1}+1, \ldots, i_{r}-2 .
$$

Under the hypothesis, $u_{2 n+1}=\bar{u}_{2 n+1}$, we will establish now that there exist at least $q^{\prime}+1$ equalities in $L_{2}^{\prime \prime}$

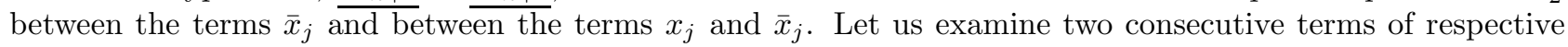
indices $t_{i}$ and $t_{i+1}$ in $L_{2}^{\prime \prime}$.

- Suppose that $t_{i+1}=t_{i}+1$, we have in this case $\bar{x}_{t_{i+1}}=\bar{z}_{t_{i}}$;

- if $t_{i+1}>t_{i}+1$, the term $\left(\bar{x}_{t_{i}+1}, \bar{u}_{t_{i}+1}, \bar{z}_{t_{i}+1}, \bar{y}_{t_{i}+1}\right)$ was removed because:

- it is is equal to an element of $L_{1}$ and consequently there exists $j \in\left\{i_{1}, i_{1}+1, \ldots, i_{r}-1\right\}$ with $j \neq t_{i}+1$ such that $\bar{x}_{t_{i}+1}=x_{j}$, from where $\bar{z}_{t_{i}}=x_{j}$;

- or it is equal to an element of list $L_{2}^{\prime \prime}$ and consequently there exists $j \in\left\{t_{i+1}, \ldots, t_{q^{\prime}}\right\}$ such that $\bar{x}_{t_{i}+1}=\bar{x}_{j}$ and consequently $\bar{z}_{t_{i}}=\bar{x}_{j}$.

To these $q^{\prime}-1$ equalities, we add the equality $\bar{x}_{j_{r}}=z_{i_{r}-1}$ and we will distinguish two situations:

- first, suppose that $t_{q^{\prime}}<i_{r}-1$, in this case, the term of index $t_{q^{\prime}}+1$ was removed because it is equal to an element of index $j$ in list $L_{1}$ and so $\bar{x}_{t_{q^{\prime}}+1}=x_{j}$ from which $\bar{z}_{t_{q^{\prime}}}=x_{j}$, which gives us a new equality;

- if $t_{q^{\prime}}=i_{r}-1$, we have $\bar{z}_{t_{q^{\prime}}}=\bar{x}_{i_{r}}=x_{\sigma^{-1}\left(i_{r}\right)}$.

At this point we have $i_{r}-i_{1}+q^{\prime}$ equalities between the $x_{j}$ 's, $z_{j}$ 's, $\bar{x}_{j}$ 's and $\bar{z}_{j}$ 's. Now by reasoning exactly in the same way than in the proof of lemma 1 , we can write $i_{r}-i_{1}$ equalities between the $u_{j}$ 's and the $\bar{u}_{j}$ 's. 


\subsubsection{Case where $\ell \neq 0$ :}

In this case there exist $\ell$ chains denoted by

$$
\begin{array}{cc}
\mathscr{C}_{1} & I\left(j_{1}^{1}\right), \ldots, I\left(j_{n_{1}}^{1}\right) \\
& \vdots \\
\mathscr{C}_{\ell} & I\left(j_{1}^{\ell}\right), \ldots, I\left(j_{n_{\ell}}^{\ell}\right)
\end{array}
$$

a chain being defined up to a circular permutation, we can suppose that, for $k=1, \ldots, \ell, j_{1}^{k} \neq 0$. As in the proof of Lemma 1 , we built the list $L_{1}^{\prime}$ extracted from $L_{1}$ by removing the elements of indices $j_{1}^{1}, \ldots, j_{1}^{\ell}$. We consider also the list $L_{2}^{\prime \prime \prime}$ extracted from $L_{2}$ by taking the terms of the list $L_{2}^{\prime \prime}$ (possibly empty) which is obtained starting from $L_{2}$ as explained in the case $\ell=0$ and by adding the terms of indices $j_{n_{1}}^{1}, \ldots, j_{n_{\ell}}^{\ell}$.

Now as in the proof of Lemma 1 Section 4.1.2, we obtain, by the consideration of consecutive elements in $L_{1}^{\prime}$, card $L_{1}^{\prime}+\ell-1$ equalities between the $x_{i}$ 's, $z_{i}$ 's, $\bar{x}_{i}$ 's and $\bar{z}_{i}$ 's. Notice that in this case we cannot have an equality like $z\left(i_{r}\right)=x\left(s_{1}^{\prime}\right)$ because $\left(x_{0}, \underline{u_{2 n+1}}\right)$ does not belong to $\mathscr{P}_{f}^{s_{1}}$ but as compensation, we have the equality $\bar{x}_{j_{r}}=z_{i_{r}-1}$. Then we can obtain also card $L_{2}^{\prime \prime \prime}-\ell$ equalities concerning the elements of list $L_{2}^{\prime \prime \prime}$. Finally, as in the proof of lemma 1 , we obtain card $L_{1}^{\prime}$ equalities between the $u_{i}$ 's and the $\bar{u}_{i}$ 's.

We conclude by applying the multijet transversality theorem exactly in the same way than in the conclusion of the proof of Lemma 1 .

\subsection{Proof of Lemma 3}

Let $f$ be given in $\left.\operatorname{Diff}_{\mathrm{U}}(X)\right)$, the set $\mathfrak{S}_{f}^{c}$ is obviously an open subset of $X \times X \times U^{2 n+1}$ and, since $X$ and $U$ are second countable, there exists a sequence $\left(K_{n}(f)\right)_{n \geq 1}$ of compact sets such that $\mathfrak{S}_{f}^{c}=\bigcup_{n \geq 1} K_{n}(f)$ and $K_{n}(f)$ is included in $\stackrel{\circ}{K}_{n+1}(f)$, the interior of $K_{n+1}(f)$. The vector space $C^{\infty}\left(X \times U, \mathbb{R}^{p}\right)$, equipped with the $C^{r}$ topology with $r<+\infty$, is a Banach space; in the following $C^{\infty}\left(X \times U, \mathbb{R}^{p}\right)$ is supposed to be equipped with this topology. We define the representation $\rho$

$$
\rho: C^{\infty}\left(X \times U, \mathbb{R}^{p}\right) \longrightarrow C^{\infty}\left(\mathfrak{S}_{f}^{c},\left(\mathbb{R}^{p}\right)^{2 n+1}\right)
$$

through the evaluation mapping :

$$
\begin{array}{rlr}
\operatorname{ev}_{\rho}: C^{\infty}\left(X \times U, \mathbb{R}^{p}\right) \times \mathfrak{S}_{f}^{c} & \longrightarrow & \left(\mathbb{R}^{p}\right)^{2 n+1} \\
\left(h, x_{0}, \bar{x}_{0}, \underline{u_{2 n+1}}\right) & \longmapsto & \left(h\left(x_{0}, u_{0}\right)-h\left(\bar{x}_{0}, u_{0}\right), \ldots,\right. \\
& & \left.h\left(f^{2 n}\left(x_{0}, \underline{u_{2 n}}\right), u_{2 n}\right)-h\left(f^{2 n}\left(\bar{x}_{0}, \underline{u_{2 n}}\right), u_{2 n}\right)\right) .
\end{array}
$$

Consider the submanifold $W=\{0\}$ of $\left(\mathbb{R}^{p}\right)^{2 n+1}$, its codimension is equal to $p(2 n+1)$ which is greater than $2 n+m(2 n+1)$ the dimension of $\mathfrak{S}_{f}^{c}$, hence to say that $\rho_{h}$ is transverse to $W$ is equivalent to say that $\rho_{h}\left(x_{0}, \bar{x}_{0}, \underline{u_{2 n+1}}\right) \neq 0$ for every $\left(x_{0}, \bar{x}_{0}, \underline{u_{2 n+1}}\right)$ in $\mathfrak{S}_{f}^{c}$ or, equivalently, that $\Theta_{2 n+1}^{f, h}\left(x_{0}, \underline{u_{2 n+1}}\right) \neq \Theta_{2 n+1}^{f, h}\left(\bar{x}_{0}, u_{2 n+1}\right)$. We will first prove the existence of a residual (for the $C^{r}$ topology) set $E^{r}$ in $C^{\infty}\left(\overline{X \times U}, \mathbb{R}^{p}\right)$ such that if the mapping $h$ is in $E^{r}, \rho_{h}$ is transverse to $W$.

In order to prove the existence of the sets $E_{r}$ we will apply the Abraham theorem (Th. 3) with $\mathcal{A}=$ $C^{\infty}\left(X \times U, \mathbb{R}^{p}\right), X=\mathfrak{S}_{f}^{c}$ and $Y=\left(\mathbb{R}^{p}\right)^{2 n+1}$. Clearly the three first hypotheses in the statement of this theorem are satisfied and we will just prove that $\operatorname{ev}_{\rho} \pitchfork W$, to this end it is sufficient to prove that $\operatorname{ev}_{\rho}$ is a submersion. First, we write the expression of $\operatorname{dev}_{\rho}$, the differential application of $\operatorname{ev}_{\rho}$ at the point $a=\left(h, x_{0}, \bar{x}_{0}, \underline{u_{2 n+1}}\right) \in$ $C^{\infty}\left(X \times U, \mathbb{R}^{p}\right) \times \mathfrak{S}_{f}^{c}:$

$$
\left(\operatorname{dev}_{\rho}\right)_{a} \cdot\left(h, \xi_{0}, \bar{\xi}_{0}, \eta_{0}, \ldots, \eta_{2 n}\right)=\left(\psi_{0}, \ldots, \psi_{2 n}\right)
$$


with

$$
\begin{aligned}
\psi_{i}= & h\left(f^{i}\left(x_{0}, \underline{u_{i}}\right), u_{i}\right)-h\left(f^{i}\left(\bar{x}_{0}, \underline{u_{i}}\right), u_{i}\right)+d_{1} h_{0}\left(f^{i}\left(x_{0}, \underline{u_{i}}\right), u_{i}\right) \cdot \xi_{0}-d_{2} h_{0}\left(f^{i}\left(\bar{x}_{0}, \underline{u_{i}}\right), u_{i}\right) \cdot \bar{\xi}_{0} \\
& +d_{3}^{i} h_{0}\left(f^{i}\left(x_{0}, \underline{u_{i}}\right), u_{i}\right) \cdot\left(\eta_{0}, \ldots, \eta_{i}\right)-d_{3}^{i} h_{0}\left(f^{i}\left(\bar{x}_{0}, \underline{u_{i}}\right), u_{i}\right) \cdot\left(\eta_{0}, \ldots, \eta_{i}\right)
\end{aligned}
$$

for $i=0, \ldots, 2 n$, the notations $d_{1}, d_{2}$ and $d_{3}^{i}$ standing for the partial derivatives at $x_{0}, \bar{x}_{0}$ and $u_{0}, \ldots, u_{i}$ respectively. Putting $\xi_{0}=0, \bar{\xi}_{0}=0$ and $\eta_{i}=0$ for $i=0, \ldots, 2 n$, the expression of $d \mathrm{ev}_{\rho}$ at $a$ becomes:

$$
\left(\operatorname{dev}_{\rho}\right)_{a} \cdot(h, 0 \ldots, 0)=\left(h\left(x_{0}, u_{0}\right)-h\left(\bar{x}_{0}, u_{0}\right), \ldots, h\left(f^{2 n}\left(x_{1}, \underline{u_{2 n}}\right), u_{2 n}\right)-h\left(f^{2 n}\left(\bar{x}_{2}, \underline{u_{2 n}}\right), u_{2 n}\right)\right) .
$$

To show that $\left(\operatorname{dev}_{\rho}\right)_{a}$ is onto, it is enough to show that, for every $\left(W_{0}, \ldots, W_{2 n}\right)$ in $\left(\mathbb{R}^{p}\right)^{2 n+1}$, there exists $h$ in $C^{\infty}\left(X \times U, \mathbb{R}^{p}\right)$ such that the following equalities are satisfied:

$$
\left\{\begin{array}{cll}
\left.h\left(x_{0}, u_{0}\right)-h\left(\bar{x}_{0}, \bar{u}_{0}\right)\right) & W_{0} \\
\vdots & & \vdots \\
h\left(f^{2 n}\left(x_{0}, \underline{u_{2 n}}\right), u_{2 n}\right)-h\left(f^{2 n}\left(\bar{x}_{0}, \underline{u_{2 n}}\right), u_{2 n}\right) & = & W_{2 n} .
\end{array}\right.
$$

Consider the two following lists

and

$$
L_{1}:\left(x_{0}, u_{0}\right), \ldots,\left(f^{2 n}\left(x_{0}, \underline{u_{2 n}}\right), u_{2 n}\right)
$$

$$
L_{2}:\left(\bar{x}_{0}, u_{0}\right), \ldots,\left(f^{2 n}\left(\bar{x}_{0}, \underline{u_{2 n}}\right), u_{2 n}\right)
$$

since $\left(x_{0}, \bar{x}_{0}, u_{2 n+1}\right)$ belongs to $\mathfrak{S}_{f}^{c}$, we can suppose without loss of generality that $\left(x_{0}, \underline{u_{2 n+1}}\right)$ is not a periodic point of $f$ and so, the elements of list $L_{1}$ are mutually distinct. However, there can exist equalities between the terms of list $L_{2}$ and between terms of $L_{1}$ and $L_{2}$. If we do not take into account the order of the elements, list $L_{2}$ can be written as $\left(a_{1}, \ldots, a_{n^{\prime}}, b_{1}, \ldots, b_{n^{\prime \prime}}\right)$, with $n^{\prime}+n^{\prime \prime}=2 n+1, a_{1}, \ldots, a_{n^{\prime}} \in L_{1}$ and $b_{1}, \ldots, b_{n^{\prime \prime}} \notin L_{1}$. We will show the existence of a function $h$ taking given values at points $\left(x_{i}, u_{i}\right)$ and such that $h\left(b_{1}\right)=\ldots=h\left(b_{n^{\prime \prime}}\right)=0$.

Consider the following system with the $p$-dimensional unknowns $\alpha_{0}, \ldots, \alpha_{2 n}$ given in $\left(\mathbb{R}^{p}\right)^{2 n+1}$

$$
\left\{\begin{array}{c}
\alpha_{0}-\sum_{j=0}^{2 n} \varepsilon_{0, j} \alpha_{j}=W_{0} \\
\vdots \\
\alpha_{2 n}-\sum_{j=0}^{2 n} \varepsilon_{2 n, j} \alpha_{j}=W_{2 n}
\end{array}\right.
$$

where

$$
\varepsilon_{i, j}= \begin{cases}1 & \text { if }\left(\bar{x}_{i}, u_{i}\right)=\left(x_{j}, u_{j}\right) \\ 0 & \text { otherwise. }\end{cases}
$$

Notice that, due to the fact that $\left(x_{0}, \bar{x}_{0}, \underline{u_{2 n+1}}\right)$ is not permutable, if we take two sets of indices $\left\{i_{1}, \ldots, i_{p}\right\}$, mutually distinct, and $\left\{j_{1}, \ldots, j_{p}\right\}$ in $\{0, \ldots, 2 n\}$ such that $\sigma\left(i_{k}\right)=j_{k}$ with $\sigma$ a permutation of $\{0, \ldots, 2 n\}$ and $k=1, \ldots, p$, we cannot have $\left(\varepsilon_{i_{1}, j_{1}}, \ldots, \varepsilon_{i_{p}, j_{p}}\right)=(1, \ldots, 1)$. Notice also that, since $x_{i} \neq \bar{x}_{i}, \varepsilon_{i, i}=0$.

We will consider the matrix $A$ associated with this linear system (10)

$$
A=\left(\begin{array}{ccccc}
I_{p} & -\varepsilon_{0,1} I_{p} & -\varepsilon_{0,2} I_{p} & \ldots & -\varepsilon_{0,2 n} I_{p} \\
-\varepsilon_{1,0} I_{p} & I_{p} & -\varepsilon_{1,2} I_{p} & \ldots & -\varepsilon_{1,2 n} I_{p} \\
\ldots \ldots \ldots \ldots \ldots \ldots \ldots \ldots \ldots \ldots \ldots \ldots \ldots \ldots \ldots \ldots & \ldots \ldots \ldots \ldots \\
-\varepsilon_{2 n, 0} I_{p} & -\varepsilon_{2 n, 1} I_{p} & -\varepsilon_{2 n, 2} I_{p} & \ldots & I_{p}
\end{array}\right)
$$


where $I_{p}$ denote the $p$-dimensional identity matrix. We will show that $\operatorname{det} A=1$, it is well known that the determinant of $A$ can be expressed as the $p$ th power of the determinant of the matrix

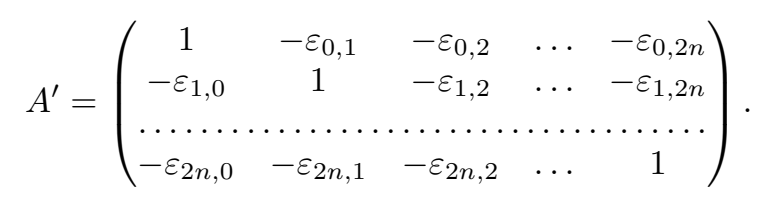

Now, we have

$$
\operatorname{det} A^{\prime}=\sum_{\sigma \in \mathfrak{S}_{2 n+1}} \varepsilon_{\sigma} a_{\sigma(0), 0} \ldots a_{\sigma(2 n), 2 n}
$$

where $\mathfrak{S}_{2 n+1}$ denotes the set of permutations of $\{0, \ldots, 2 n\}, \varepsilon_{\sigma}$, the sign of permutation $\sigma$ and the $a_{i, j}$ 's are the terms of matrix $A^{\prime}$. If $\sigma$ is the identity permutation, we have

$$
\varepsilon_{\sigma} a_{\sigma(0), 0} \ldots a_{\sigma(2 n), 2 n}=1
$$

If $\sigma$ is different from identity let $\left\{k_{1}, \ldots, k_{p}\right\}$ be the set of fixed points of $\sigma$ and put $\left\{i_{1}, \ldots, i_{q}\right\}=\{0, \ldots, 2 n\} \backslash$ $\left\{k_{1}, \ldots, k_{p}\right\}$, this last set is non empty and, letting $j_{k}=\sigma\left(i_{k}\right)$, we have

$$
\varepsilon_{\sigma} a_{\sigma(0), 0} \ldots a_{\sigma(2 n), 2 n}=\varepsilon_{\sigma}(-1)^{q} \varepsilon_{j_{1}, i_{1}} \ldots \varepsilon_{j_{q}, i_{q}}
$$

which is zero because $\left(\varepsilon_{j_{1}, i_{1}}, \ldots, \varepsilon_{j_{q}, i_{q}}\right) \neq(1, \ldots, 1)$. So we proved that $\operatorname{det} A^{\prime}=1$.

The consequence of this computation is that system $(10)$ has a solution $\left(\alpha_{0}, \ldots, \alpha_{2 n}\right)$, for these values, we can find a mapping $h$ in $C^{\infty}\left(X \times U, \mathbb{R}^{p}\right)$ such that $h\left(x_{i}\right)=\alpha_{i}$ for $i=0, \ldots, 2 n$ and $h\left(b_{i}\right)=0$ for $i=1, \ldots, n^{\prime \prime}$. Clearly Such a mapping $h$ is a solution of system (9).

At this stage, we have a residual set $E^{r}$ included in $C^{\infty}\left(X \times U, \mathbb{R}^{p}\right)$ such that every mapping $h$ in $E^{r}$ is such that the pair $(f, h)$ satisfies property $\mathscr{P}_{2}$. Now, using Theorem 4 , we can see that the set

$$
U_{n}^{r}(f)=\left\{h \in C^{\infty}\left(X \times U, \mathbb{R}^{p}\right) \mid \rho_{h} \pitchfork_{x} W \text { for } x \in K_{n}(f)\right\}
$$

is open, and, since $E^{r}$ is obviously included in $U_{n}^{r}(f)$, it is also dense. Proving that the set $\mathscr{U}$ is open is a quite delicate task. First, we will be more specific about the construction of the compact sets $K_{n}(f)$ : given a sequence of compact sets $\left(J_{n}(f)\right)_{n \geq 1}$ such that

$$
\mathfrak{S}_{f}^{c}=\bigcup_{n \geq 1} J_{n}(f) \text { and } J_{n}(f)=\stackrel{\circ}{J}_{n+1}(f)
$$

we can write the set $\mathfrak{S}_{f}^{c}$ as

$$
K_{n}(f)=J_{n}(f) \cap\left\{v \in \mathfrak{S}_{f}^{c} \mid d\left(v, P_{f} \cup \mathfrak{S}_{f}\right) \geq \frac{1}{n}\right\}
$$

where $d$ is a distance compatible with the topology of $X \times X \times U^{2 n+1}$; recall also that $P_{f} \cup \mathfrak{S}_{f}$ is the set complement of $\mathfrak{S}_{f}^{c}$ in $M \times M \times U$.

Now if the parameterized diffeomorphism $f$ is closed to $f_{0}$, set $\mathfrak{S}_{f}^{c}$ is closed to $\mathfrak{S}_{f_{0}}^{c}$ and so are the sets $K_{n}(f)$ and $K_{n}\left(f_{0}\right)$ defined as above. The representation $\rho$ defined by equality $(8)$ and set $E^{r}$ depend on diffeomorphism $f$, to avoid ambiguity, in what follows, we will denote them by $\rho^{f}$ and $E^{r}(f)$. Take now $\left(f_{0}, h_{0}\right)$ in $\mathscr{U}_{n}^{r}$, there exists $m>0$ such that for every $\left(x_{0}, \bar{x}_{0}, \underline{u_{2 n+1}}\right)$ in $K_{n}\left(f_{0}\right)$,

$$
\left\|\rho_{h_{0}}^{f_{0}}\left(x_{0}, \bar{x}_{0}, \underline{u_{2 n+1}}\right)\right\| \geq m
$$


if $(f, h)$ is closed enough to $\left(f_{0}, h_{0}\right), K_{n}\left(f_{0}\right)$ is closed to $K_{n}(f)$ and we have

$$
\left\|\rho_{h}^{f}\left(x_{0}, \bar{x}_{0}, \underline{u_{2 n+1}}\right)\right\| \geq \frac{m}{2}
$$

for every pair $(f, h)$ in some neighborhood of $\left(f_{0}, h_{0}\right)$; this proves the openness of $\mathscr{U}_{n}^{r}$.

\section{Conclusion}

In this paper, we proved that, generically, a discrete-time nonlinear system is strongly observable provided that the number of outputs is greater than the number of inputs. We made the assumption that the observation function $h$ depends on the state variable $x$ and the input $u$, nevertheless the same result is true if function $h$ depends only on $x$, we will indicate briefly how this result could be proved. The outline of the proof of Theorem 5 is the same, and we have just to make the following slight modifications. If $f$ is in $\operatorname{Diff}_{\mathrm{U}}(X)$ and $h$ is in $C^{\infty}\left(X, \mathbb{R}^{p}\right)$, the notation $(f, h)$ stand for the mapping

$$
\begin{aligned}
(f, h): X \times U \times X & \longrightarrow X \times \mathbb{R}^{p} \\
(x, u, w) & \longmapsto(f(x, u), h(w)) .
\end{aligned}
$$

In the proofs of Lemmas 1 and 2, the lists to be considered have to be modified as follows:

$$
\begin{array}{ll}
L_{1} & \left(x_{0}, u_{0}, w_{0}, z_{0}, y_{0}\right), \ldots,\left(x_{s_{1}-1}, u_{s_{1}-1}, w_{s_{1}-1}, z_{s_{1}-1}, y_{s_{1}-1}\right) \\
L_{2} & \left(\bar{x}_{0}, \bar{u}_{0}, \bar{w}_{0}, \bar{z}_{0}, \bar{y}_{0}\right), \ldots,\left(\bar{x}_{s_{1}-1}, \bar{u}_{s_{1}-1}, \bar{w}_{s_{1}-1}, \bar{z}_{s_{1}-1}, \bar{y}_{s_{1}-1}\right)
\end{array}
$$

and we work under the assumption $w_{0}=x_{0}, \ldots, w_{s_{1}-1}=x_{s_{1}-1}$ and $\bar{w}_{0}=\bar{x}_{0}, \ldots, \bar{w}_{s_{1}-1}=\bar{x}_{s_{1}-1}$. These equalities have to be added to the equalities established in the first parts of the proofs of Lemmas 1 and 2 . Now, in the application of the multijet transversality theorem, we consider mapping from $(X \times U \times X)^{\left(d_{1}+d_{2}\right)}$ to $X \times U \times X \times X \times \mathbb{R}^{p}$, thanks to these extra equalities, the codimension of submanifold $W$ in $X \times U \times X \times X \times \mathbb{R}^{p}$ is greater than the dimension of $(X \times U \times X)^{\left(d_{1}+d_{2}\right)}$ which allows us to conclude as in the proofs of Lemmas 1 and 2. Nothing has to be changed in the proof of Lemma 3. Finally we shall examine the assumptions of our main result. First, we claim that it is not possible to obtain the same result if we relax the hypothesis " $f_{u}$ is a diffeomorphism". In [11] indeed, we provide the following counterexample: $\mathbb{T}^{n}$ denoting the $n$-dimensional torus, consider the mapping

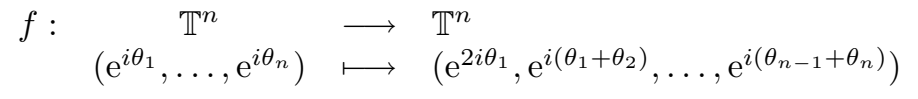

together with

$$
h: \underset{\mathbb{T}^{n}}{\left(\mathrm{e}^{i \theta_{1}}, \ldots, \mathrm{e}^{i \theta_{n}}\right)} \stackrel{\longrightarrow \mathbb{R}^{n}}{\left(\sin \theta_{1}, \ldots, \sin \theta_{n}\right) .}
$$

Mapping $f$ is smooth but is not a diffeomorphism and the (uncontrolled) discrete-time system defined on $\mathbb{T}^{n}$ by $f$ and $h$ is unobservable; moreover in the above-mentioned paper, we prove the existence around the pair $(f, h)$ of an open set in $C^{\infty}\left(X, X \times \mathbb{R}^{n}\right)$ constituted by unobservable discrete-time systems.

Now is it possible to relax the assumption about the dimensions of the input and output spaces? In $[4,6]$, the authors have proved that if $p=1$ and $\operatorname{dim} U \geq 1$, a continuous-time uniformly infinitesimally observable system can be put locally in observability canonical form. The non genericity of this canonical form leads us to think that, if $p \leq \operatorname{dim} U$, observability is no more a generic property. As a matter of fact, we provide below a simple example illustrating this assertion. As usual, we denote by $S^{1}$ the circle $S^{1}=\{z \in \mathbb{C}|| z \mid=1\}$ We take $X=S^{1}, U=S^{1}$ and we consider the following mappings

$$
\begin{aligned}
& f_{0}: S^{1} \times S^{1} \longrightarrow S^{1} \\
& \left(\mathrm{e}^{i \theta}, u\right) \longmapsto u \mathrm{e}^{i \theta}
\end{aligned}
$$


and

$$
\begin{aligned}
h_{0}: & S^{1} \times S^{1} \longrightarrow \mathbb{R} \\
& \left(\mathrm{e}^{i \theta}, \mathrm{e}^{i \alpha}\right) \\
& \longmapsto \sin (\theta-\alpha)
\end{aligned}
$$

which are smooth, $f_{0}$ being such that $\left(f_{u}\right)_{0}$ is a diffeomorphism of $S^{1}$ for every $u$. We shall prove "by hand" that one can find around the pair $\left(f_{0}, h_{0}\right)$ an open neighborhood constituted by unobservable dynamical systems $(f, h)$. First notice that, letting

$$
\begin{array}{lll}
x_{0}=1 & \bar{x}_{0}=-1 & u_{0}=u_{1}=u_{2}=1 \\
x_{1}=f_{0}\left(x_{0}, u_{0}\right) & x_{2}=f_{0}\left(x_{1}, u_{1}\right) & \\
\bar{x}_{1}=f_{0}\left(\bar{x}_{0}, u_{0}\right) & \bar{x}_{2}=f_{0}\left(\bar{x}_{1}, u_{1}\right) &
\end{array}
$$

we have $h_{0}\left(x_{i}, u_{i}\right)=h_{0}\left(\bar{x}_{i}, u_{i}\right)$ for $i=0,1,2$. If the function $h$ is closed enough to $h_{0}$ (in the sense of $C^{0}$ topology), the expression $h\left(1, \mathrm{e}^{i \alpha}\right)-h\left(-1, \mathrm{e}^{i \alpha}\right)$ is closed to $h_{0}\left(1, \mathrm{e}^{i \alpha}\right)-h_{0}\left(-1, \mathrm{e}^{i \alpha}\right)=-2 \sin \alpha$ and so it changes its sign as $\alpha$ varies from $-\pi / 4$ to $\pi / 4$, hence there exists $v_{0}=\mathrm{e}^{i \alpha_{0}}$ such that $h\left(1, \mathrm{e}^{i \alpha_{0}}\right)=h\left(-1, \mathrm{e}^{i \alpha_{0}}\right)$. Now if the parameterized diffeomorphism $f$ is closed enough to $f_{0}, f\left(1, v_{0}\right)$ and $f\left(-1, v_{0}\right)$ are closed to $f_{0}\left(1, v_{0}\right)$ and $f_{0}\left(-1, v_{0}\right)$ and the expression $h\left(f\left(1, u_{0}\right), \mathrm{e}^{i \alpha}\right)-h\left(f\left(-1, u_{0}\right), \mathrm{e}^{i \alpha}\right)$ is closed to

$$
h_{0}\left(f_{0}\left(1, u_{0}\right), \mathrm{e}^{i \alpha}\right)-h_{0}\left(f_{0}\left(-1, u_{0}\right), \mathrm{e}^{i \alpha}\right)=2 \sin \left(\alpha_{0}-\alpha\right)
$$

which changes its sign as $\alpha$ varies in an open interval containing $\alpha_{0}$, so there exists $v_{1}=\mathrm{e}^{i \alpha_{1}}$ such that $h\left(f\left(1, v_{0}\right), v_{1}\right)=h\left(f\left(-1, v_{0}\right), v_{1}\right)$. A similar reasoning proves the existence of $v_{2}=\mathrm{e}^{i \alpha_{2}}$ such that $h\left(f\left(f\left(1, v_{0}\right), v_{1}\right), v_{2}\right)=$ $h\left(f\left(f\left(-1, v_{0}\right), v_{1}\right), v_{2}\right)$. Thus we have proved the existence of an neighborhood of the pair $\left(f_{0}, h_{0}\right)$, open for the $C^{0}$-topology (and hence for the $C^{\infty}$-topology) such that, for every pair $(f, h)$ in this neighborhood, there exist $v 0, v_{1}$ and $v_{2}$ such that

$$
h\left(1, v_{0}\right)=h\left(-1, v_{0}\right) \quad h\left(z_{1}, v_{1}\right)=h\left(\bar{z}_{1}, v_{1}\right) \quad h\left(z_{2}, v_{2}\right)=h\left(\bar{z}_{2}, v_{2}\right)
$$

where

$$
z_{1}=f\left(1, v_{0}\right) \quad \bar{z}_{1}=f\left(-1, v_{0}\right) \quad z_{2}=f\left(z_{1}, v_{1}\right) \quad \bar{z}_{2}=f\left(\bar{z}_{1}, v_{1}\right)
$$

which means that the observed dynamical system defined by the pair $(f, h)$ on $S^{1}$ is not observable.

\section{REFERENCES}

[1] R. Abraham and J.W. Robbin, Transversal Mappings and Flows. W. A. Benjamin, New York (1967).

[2] D. Aeyels, Generic observability of differentiable systems. SIAM J. Control Optim. 19 (1981) 595-603.

[3] J.-P. Gauthier, H. Hammouri and I. Kupka, Observers for nonlinear systems. In Proc. of the IEEE 30th CDC (1991) 1483-1489.

[4] J.-P. Gauthier and I. Kupka, Observability and observers for nonlinear systems. SIAM J. Control Optim. 32 (1994) 975-994.

[5] J.-P. Gauthier and I. Kupka, Observability for systems with more outputs than inputs and asymptotic observers. Math. Zeitschrift 223 (1996) 47-78.

[6] J.P. Gauthier and I. Kupka, Deterministic observation: theory and applications. Cambridge: Cambridge University Press (2002).

[7] M. Golubitsky and V. Guillemin, Stable Mappings and Their Singularities. Springer-Verlag, New York (1986).

[8] J. Stark, Delay embedding for forced systems - I. Deterministic forcing. J. Nonlinear Sci. 9 (1999) 255-332.

[9] J. Stark, D.S. Broomhead, M.E. Davies and J. Huke, Delay embedding for forced systems - II. Stochastic forcing. J. Nonlinear Sci. (to appear).

[10] F. Takens, Detecting strange attractors in turbulence. No. 898 in Lect. Notes Math. Springer-Verlag, Coventry (1981).

[11] J.-C. Vivalda, On the genericity of the observability of uncontrolled discrete nonlinear systems. SIAM J. Control Optim. 42 (2003). 